Systems and Signal Processing

Elsevier Editorial System(tm) for Mechanical Manuscript Draft

Manuscript Number: MSSP16-1379R2

Title: Constrained Non-linear Multi-objective Optimization of Preventive Maintenance Scheduling for Offshore Wind Farms

Article Type: Full Length Article

Keywords: Reliability, Maintenance Scheduling; Cost Parameters; Offshore Wind Farms; Multi-objective Programming

Corresponding Author: Professor Michael Beer, Dr.-Ing.

Corresponding Author's Institution: Leibniz University Hannover

First Author: Shuya Zhong, PhD

Order of Authors: Shuya Zhong, PhD; Athanasios A Pantelous, PhD; Michael Beer, Dr.-Ing.; Jian Zhou, PhD 
Faculty of Civil Engineering and Geodetic Science

Institute for

Risk and Reliability

Professor John Mottershead

Editor in Chief

Journal of

Mechanical Systems and Signal Processing

\section{SUBMISSION OF THE SECOND REVISION: MANUSCRIPT MSSP16-1379}

Dear Professor Mottershead,

we are herewith submitting the second revision of the manuscript MSSP16-1379

"Constrained Non-linear Multi-Objective Optimization of Preventive Maintenance Scheduling for Offshore Wind Farms"

for possible publication in the Journal of Mechanical Systems and Signal Processing.

We would like to express our sincere appreciation to the reviewers for the constructive comments to improve our contribution. Specifically, we would like to thank reviewer \#2 for highlighting important issues. The reviewer's comments have been taken into careful consideration, and the suggested changes have been implemented accordingly.

Moreover, we looked all paragraphs and phrases, and we improved the manuscript comprehensively on typos, spelling mistakes and editing. The major changes are highlighted in blue color in the text. We omitted to color in blue the minor changes.

We would be very pleased if you could please be so kind as to consider this submission for further processing.

We herewith declare that this manuscript is the authors' original work and has not been published nor has it been submitted simultaneously elsewhere. All authors have checked the manuscript and have agreed to the submission.

If you have any question or if you need any additional information please do not hesitate to contact me.

Sincerely,

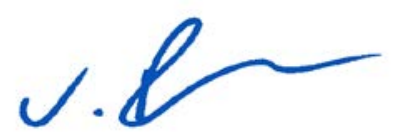

Michael Beer
Prof. Dr.-Ing.

Michael Beer

Head of Institute

Tel. +495117625981

Fax +495117624756

eMail:

beer@irz.

uni-hannover.de

September 18, 2017

Visitors Address:

Callinstraße 34

www.irz.uni-hannover.de

Secretariat:

Tel. +49 5117625981

Fax +49 5117624756

office@irz.uni-hannover.de
30167 Hannover 


\section{Response to the reviewers}

Reviewer \#1: The reviewer did not request any further modification or change.

\section{Reviewer \#2:}

1. The authors have addressed only some minor point raised in the review and have failed in addressing the main point related to the meaning of the "attainment exponent".

The application of this exponent to the original reliability index (power reserve ratio) can shift the reliability towards high values (optimistic attitude) or low values (pessimistic 1 attitude). In Section 6.2, different Pareto fronts are obtained as a result of different attitudes. The practical implication and use of the introduction of the attainment exponent is yet not evident to me. For example (Fig 3 and Table 4), if the decision-maker believes that the maintenance will always have a positive effect (optimistic attitude), the total "anticipated" reliability will be obviously large. The costs are in the range [7.1 , 8.9]. The same hold if the decision-maker believes that the maintenance has a neutral effect (waitand-see attitude). On the other hand, if the decision-maker believes that the maintenance will have a positive effect initially but will not be able to contrast the effects of the harsh environment in the long run (rational), the total "anticipated" reliability is in a mid range, but the costs are in the range $[6.9,8.6]$.

Based on the above, the authors conclude that "the fully rational attitude is more appropriate for decision-makers to hold because it does not only offer more diverse options, but also makes the results more reasonable and effective." Yet, this is clearly not the case. The Optimistic, Wait-and-See and Pessimistic 2 attitudes offer better reliability in the same cost range.

In their current presentation, the obtained results do not justify the practical application or the benefits of considering the attainment exponent. 
Answer. We would like to thank the reviewer for his concerns, and the opportunity he gave us to improve and motivate better our contribution.

First, mathematical speaking, with the proposed "attainment exponent", which is a curvature parameter, we want to give another dimension to the reliability index. Actually, in this paper, we recommend for the very first time according to the authors' knowledge the use of an isoelastic function (or in another word, the use of a power utility) to model the behavioral attitude of our treatment. The isoelastic utility function is a special case of the hyperbolic absolute risk aversion (HARA) utility functions, and is used in analyses either including or not including the underlying risk. For more details, you can consult:

- Arrow, K. J. (1965). "The theory of risk aversion". Aspects of the Theory of Risk Bearing. Helsinki: Yrjo Jahnssonin Saatio. Reprinted in: Essays in the Theory of Risk Bearing. Chicago: Markham. 1971. pp. 90-109.

- Pratt, J. W. (1964). "Risk aversion in the small and in the large". Econometrica. 32 (1-2): 122-136.

(among many other papers \& celebrated books)

Second, the curvature parameter (or "attainment exponent”) has been used extensively in decision making theory to express the utility in terms of satisfaction or attitude or some other behavioural aspects that a decision-maker is concerned with. For instance, in classical economics, the curvature controls the preference over some set of goods (including services: something that satisfies human wants). In another words, it models satisfaction experienced by the consumer or performance experienced by the end-user of a good.

Thus, in our paper, we wanted to implement this concept and to increase the degree of flexibility out of the reliability index. For instance, we believe that the attitude/preference of the decision maker is not the same when the local power grid has been affected by a catastrophe, such as the IRMA case recently, and the only alternative source of energy is available through the offshore wind farm. The magnitude of reliability index of the offshore wind farm is getting higher and more important to the policy makers as well to 
consumers and government. Similarly, the attitude of the decision maker is downgraded, if there is extra capacity in the market or if the consumers' price is getting lower based on some social-economic-political interventions. Several other scenarios can be considered; however this is far beyond the scope of this present study.

Furthermore, perhaps, it would be helpful to underline that this concept is an important underpinning of rational choice theory in economics and game theory.

Empirically speaking, and we will agree with your concerns, we cannot directly measure benefit, satisfaction or happiness from a good or service. However, economists and mathematicians instead have devised ways of representing and measuring utility in terms of measurable economic choices. The isoelastic case (which has been used in the present paper) is the simplest one. More advanced structures for capturing more complicated behavioral aspects can be considered in sequence papers.

Of course, you can always assume that the "attainment exponent” is equal to 1 , and the behavioural attitude can be neglected completely in the optimization process.

Some misleading parts of the previous version have been eliminated. Thank you that you pointed out to us. Also, we added a few more clarifications, see for instance:

"Thus, in what follows, we test some predefined behavioral attitudes of the decision-makers. Obviously, the proposed four categories, "fully rational", "optimism biased", "waitand-see attitudes" and "pessimism biased" are initiating and inspiring, rather than exhaustive and conclusive for the research on maintenance, and more generally speaking, in the behavioral approach of the reliability index and our multi-objective constrained optimization problem. So, let us define the four categories of attitudes:” (page 11)

Additionally, we tried to interpret more clearly that why the fully rational attitude is better comparing with the other two, and the benefit of adopting the attainment exponent as follows, "It is apparent from Fig. 2 and more precisely from Table 4 that results on the basis of the fully rational attitude (yellow asterisks in Fig. 2 and 2nd row in Table 4) have the best spread of Pareto-optimal solutions, which can provide much wider and more 
distinguishable choices both on system reliability (changes from 0.684 to 0.845 ) and maintenance cost (from \{leuro\}6.939m to \{leuro\}8.600m) directions for trading-off and supporting the decision-making. Although solutions with optimistic, wait-and-see and pessimistic (\$s_t=2\$) attitudes achieve extremely high values of reliability, their solution sets contain a few gaps and their spreads are relatively narrow and partial on the reliability axis. Moreover, solutions with the pessimistic attitude (\$s_t>1\$) form good spreads on both of the reliability and the cost axes, but their values of reliability are relatively too low (even the upper bound $\$ \mid \operatorname{mbox}\{$ Reliability\}=0.553\$). Hence, we can conclude that the fully rational attitude seems more appropriate for decision-makers to hold because it not only offers more diverse options, but also makes the results more reasonable and effective.

Thus, in the following analyses, we will primarily focus on the multi-objective optimization model with attainment exponents setting based on the fully rational attitude. Obviously, this is not an exhaustive and conclusive way, as different decision-makers can take an alternative strategy as the most preferable one. Actually, the model flexibility is one of the main advantage of our treatment".

Moreover, the paper presentation has not been improved as requested. The itemized comments below may help in this task.

\section{Itemized comments}

2. Title: Why do the authors mention "Constrained"? Is this a specialty of their problem? Aren't all the optimization problems constrained?

Answer. Indeed, there is significant literature about unconstrained optimization problems. See for instance, the following recent books, and their chapters.

- Forst, Wilhelm, and Dieter Hoffmann. Optimization-Theory and Practice. Springer Science \& Business Media, 2010.

- Bertsekas, Dimitri P. Constrained optimization and Lagrange multiplier methods. Academic press, 2014. 
On the contrary, in optimization theory jargon, it is common not to use the term "constrained" if the optimization is unconstrained.

Furthermore, since we especially design the constraints applicable to the offshore wind energy scenario for our problem, the "constrained" in the title is used to highlight this contribution as well.

3. Page 3: "...the deviation of the net reserve for a uniformly distributed reserve margin." This sentence is unclear. Why "for"?

Answer. Thank you. We revise the above sentence as "the second one indicates the deviation of the net power reserve, i.e., the reserve margin".

4. Page 3: "...quantified the reliability by the sum of the squares of net reserves (SSR)." The authors should refer to Section II of the paper "Egan, G.T., DilIon, T.S. and Morsztyn, K. (1976) An experimental method of determination of optimal maintenance schedules in power systems using branch-and-bound technique, IEEE Transactions on Systems, Man and Cybernetics 6, 538-547". Reliability is not "quantified by the SSR"; the minimization of the SSR objective may contribute to the maximization of the reliability.

Answer. We highly appreciate that the reviewer points out the pioneer work of the reliability objective definition for the maintenance scheduling problems in the power systems. Therefore, we revise the sentence as "Egan et al. (1976) first proposed that the minimization of the sum of the squares of the reserves (SSR) would prevent the large variations in the net power reserves of each time period, which means the maximization of the reliability".

5. Page 3, 8, 9, and others: The authors should rename the "power storage ratio" mentioned in the paper into "power reserve ratio". The use of the word "storage" is connected to storage systems and batteries, but not to the allocation of power reserves as it is investigated here.

Answer. Thank you. We revise the "power storage ratio" to "power reserve ratio". 
6. Page 3: "...to get closer to its actual attainment level." This phrase is convoluted; it adds little insight. The authors should clarify what they practically mean by this phrase. 7. Page 3: "...actual power storage ratio...". What is the definition of the actual power storage ratio? Is it the same as the definition of "the net reserve being divided by the gross reserve", which is presented in [4]? The authors should clearly define this concept and keep the same name for it throughout the paper.

8. Page 3: "...we think that another factor, the sustainable ability, which reflects the real power output condition, is of equal importance." The authors should define the concept of "sustainable ability" at this point, in which it is first mentioned. Postponing its introduction delays understanding of the methodology.

9. Page 3-4: "...we are motivated by this idea to propose a novel non-linear definition of the reliability within both of the two considerations..." The meaning of "within both of the two considerations" is unclear. This phrase should be checked for clarity.

10. Page 4: "...attainment exponent...". The authors should define this concept here when it is first introduced. Why delaying its definition and hence the presentation of their novel methodology?

Answer. The above five questions (6 - 10) are mentioned for the third paragraph of Section 2.1. We refine the whole paragraph carefully by integrating the suggestions of the reviewer as follows, "In our paper, we will adjust the first type of the conventional reliability criterion in the PM scheduling of offshore wind farms to model the behavioral attitude of our treatment. As only the customer power demand satisfaction delineated by the power reserve ratio has been studied in the previous definitions from the demand perspective, here the reliability criterion can be better depicted if the decision-maker preferences are also taken into account over a set of choices or attitudes. Moreover, in offshore wind farms, the particularly complex and variable marine environment contributes to the unstable effects of the maintenance and degeneration on the real power reserve which may not have such significant influence on other kinds of power plants 
Icite\{Schiermeier2013\}. Therefore, another factor, the system sustainability, which means the sustainable capability of reserving the power under the combined impacts of the maintenance work and the system degradation in each time period, is of equal importance to be considered in the reliability frame. It can reflect the actually attained power reserve ratio by exponentially adjusting the estimated power reserve ratio. Thus, we propose a novel non-linear definition of the reliability with both of the demand and supply side regards by introducing what we call the "attainment exponent"Ifootnote\{ This can also be seen a curvature parameter in the reliability, see Section 4.2.1. \}, so as to describe the decision-maker's preferences, the power demand satisfaction and the system sustainability simultaneously.”.

11. Page 4: "the power production, maintenance, start-up, fixed, variable, opportunity, compensation, and failure costs." The authors should provide next to each of these eight cost items one reference, in which the item is defined and used as the objective of the optimization.

Answer. Thank you. We summarize some representative works in Table 1, containing the 8-maintenance related cost composition for power systems. We have tried to make a comprehensive literature review.

12. Page 4: "...we refer to the definitions of the no-failure maintenance cost presented by Dahal et al. [7] and Dalgic et al. [10] to some degree..." and "In addition, some other cost factors particularly for wind farms are also involved in our definition as indicated by Ding and Tian [12] and Gundegjerde et al. [16], e.g., the fixed cost of sending vessels to wind farms for maintenance, the variable access cost to wind turbines, etc." The authors keep anticipating bits and pieces of the description of the proposed model without providing a formal definition. Furthermore, they partially describe the model, i.e. they use expressions as "to some degree" and "etc.". The authors should streamline the presentation of their model in one block, without early partial definitions and successive detailed presentation. As it is currently structured, Section 2 is not a literature review as claimed by the authors, 
but it also contains the partial presentation of the model, which adds little insight for the reader.

Answer. In our views, Section 2 completes the short part of our introduction and explains better our motivation part. Actually, it articulates a detailed formation of the design philosophies logically (the literature review of the present methods to develop the two objectives, the points we are inspired and the defects we will improve, as well as the innovative approaches we will develop in brief) of the reliability and cost objectives for our problem. Therefore, Section 2 is neither just a simple literature review nor a concrete model formulation. It functions as a link of the cause and effect of structuring the two criteria. Hence, since it is just matter of taste, we would like to politely ask you to remain the contents mentioned above as the same.

13. Page 5: "...the relationship between reliability and maintenance cost objectives can be deeply studied by vertical analyses and horizontal comparisons." The authors should rephrase this sentence and replace the phrases "vertical analyses" and "horizontal comparisons" for clarity.

Answer. Thank you. We revise the above sentence as "Thus, the relationship between reliability and maintenance cost objectives can be deeply studied by analyzing the tradeoffs between the two goals, as well as comparing them using the proposed as well the contrast model”.

14. Page 6: "Our problem is to arrange the maintenance of offshore wind farms containing m turbines into n time periods." and "Our aim is to allocate $m$ turbines in offshore wind farms to implement their maintenance in different time periods,..." The authors should remove this repetition occurring within one line.

Answer. Thank you. We remove the first sentence of Section 4.1 to eliminate the redundancy. 
15. Page 8: "... and simultaneously to consider the effects of the sustainability." and "the sustainable development of the system". The authors keep mentioning this idea of "sustainability" without defining it. At this stage, they have already mentioned it too many times and should have already provided a definition to guide the understanding of the reader on what they mean and propose here.

Answer. Thank you. We supplement the interpretation of the system sustainability in the third paragraph of Section 2.1. It means the sustainable capability of reserving the power under the combined impacts of the maintenance work and the system degradation in each time period. It can reflect the actually attained power reserve ratio by exponentially adjusting the estimated power reserve ratio. The proposed attainment exponent is utilized to express the system sustainability.

16. Page 8: "...the risk occurring of serious total grid breakdowns." What is the meaning of the phrase "the risk occurring of"? The authors should rephrase it.

Answer. Thank you. We revise the above phrase as "reduce the risk leading to serious grid breakdowns".

17. Page 8: "...to provide a real reflection of the customer demand satisfaction." What is the meaning of the phrase "to provide a real reflection of"? The authors should rephrase. Answer. Thank you. We revise the above sentence as "Thereby, three different possible effects emerge to provide actual achievements of the customer power demand satisfaction, i.e., the power reserve ratio”.

18. Page 8: the authors should replace "composition" by "average".

Answer. Thank you. We replace “composition” by "average”.

19. Page 9: "...lower reliability than averages." Why do the authors use the word "averages" in its plural form?

Answer. Thank you. We replace “averages” by "the average”. 
20. Page 9: "They are actually two constitutive elements..." The authors should rephrase and clarify which two elements they are referring to.

Answer. Thank you. We supplement the interpretation as "Since the electricity generated and demanded in each time period is an approximate estimate in terms of the historical data, the power reserve ratio $\$ e \_t / E \_t \$$ which has eliminated the influence of the maintenance downtime, as well as the attainment exponent \$s_t\$ reflecting the effects of decision-makers attitude, can coordinate to represent the actual achievement of the customer power demand satisfaction. The power reserve ratio and the attainment exponent are two constitutive elements of the reliability $\$ r \_t \$$ in $\$ \mid \operatorname{mbox}\{P R\} \_t \$ . ”$

21. Page 9: the authors should replace "integrating" by "averaging".

Answer. Thank you. We replace “integrating” by “averaging”.

22. Page 9: "...in which weight coefficients $1 / n . . . " .1 / n$ is actually one coefficient, so why do the authors use the plural form of the word "coefficient"?

Answer. Thank you. We replace "weight coefficients" by "the weight coefficient”.

23. Page 14: Figure 2 should be removed because it add little to the understanding of the methodology.

Answer. Thank you. It has been removed.

24. Page 20: "Transforming convenience manifests the good applicability and flexibility of Model (27a, b), so that we can declare convictively the PM scheduling model proposed in this paper is complete and reasonable." The word "convictively" seems not a good fit here. The authors should rephrase this sentence because it does sound awkward.

Answer. Thank you. We revise the above sentence as "The convenience of transforming manifests the good applicability and flexibility of Model $(27 a, b)$, so that we can declare that the PM scheduling model proposed in this paper is reasonable" 
25. Page 20: "It can be seen that this definition of the reliability maximization objective generates from another perspective that they think high system reliability means to decrease differences among net power reserves for each time period,..." The authors should rephrase this sentence.

Answer. Thank you. We revise the above sentence as "This definition of the reliability objective is generated from another perspective that the high system reliability implies the little difference among the net power reserves for each time period, namely to make full use of the electric energy and avoid power waste”.

26. Page 20: "...the weight coefficients...". Actually, this is only one coefficient resulting from the summation from 1 to $\mathrm{n}$. Therefore, the authors should use the singular form.

Answer. Thank you. We replace “coefficients” by “coefficient”.

27. Page 20: "...to compare the two definitions of the system reliability." Actually, Eq. 28 has never been proposed as definition of reliability but as an objective, whose minimization can maximize reliability. The authors should mention explicitly and clearly that the two values of reliability from Eq. 27a and from Eq. 29 are originated from two different reliability indices, which provide two different interpretations of the reliability ensured by power reserves. This remark should also be explicitly mentioned in Section 6.4 when the two reliability values are plotted in the same axes in Figure 6.

Answer. Thank you. It has been done.

28. Page 20, Eq. 29: the index $t$ in the series at the denominator should be changed into another symbol because it conflicts with the index t of the outermost series.

Answer. Thank you. It has been done.

29. Section 6.1: the authors should provide the numerical values of all the system and maintenance parameters used in the study.

Answer. Thank you. We supplement all the system and maintenance parameters in Tables. 6-10 in Appendix. 
30. Page 23, Figure 3: the correspondence between the symbols and the attitudes is not explicit. The authors should include the attitude labels in the legend.

Answer. Thank you. We supplement the attitude labels in the legend.

31. Page 24: "...it can be concluded that the fully rational attitude is more appropriate for decision-makers to hold because it does not only offer more diverse options, but also makes the results more reasonable and effective." This is clearly not the case. The Optimistic, Wait-and-See and Pessimistic 2 attitudes offer better reliability in the same cost range. The authors should explain why they have concluded that "the fully rational attitude is more appropriate".

Answer. Please, see our explanation in Question 1.

32. Page 28: "When the execution of the project begins to stabilise, the differentiation strategy (i.e., the customer-oriented strategy) is more likely to be adopted in order to satisfy customer needs for more profits." Each solutions in the Pareto front of Fig. 3 involve the optimization of the entire time horizon using the same strategy across the time horizon. The authors should explain how the decision-maker can assess the effects of changing and "adopting" different maintenance strategies during the time horizon.

Answer. Thank you. We supplement the interpretation as "The maintenance schedule solutions corresponding to a certain maintenance strategy (cost leadership, differentiation, or focus strategies) can be timely and newly obtained by implementing the model and algorithm again after constraining those wind turbines that already completed the maintenance jobs in the past periods, whenever the decision-maker determines to switch to a different strategy from the present one at any period during the time horizon".

Additionally, several new references have been added.

Also, we checked again carefully the paper about typos \& spelling mistakes. 
- A non-linear multi-objective programming model is proposed for preventive maintenance of offshore wind farms

- Maximization of system reliability and minimization of maintenance related to cost are considered simultaneously.

- The optimization is solved with a nondominated sorting genetic algorithm II. 


\title{
Constrained Non-linear Multi-objective Optimization of Preventive Maintenance Scheduling for Offshore Wind Farms
}

\author{
Shuya Zhong ${ }^{\mathrm{a}, \mathrm{f}}$, Athanasios A. Pantelous ${ }^{\mathrm{b}, \mathrm{c}, \mathrm{f}}$, Michael Beer ${ }^{\mathrm{b}, \mathrm{d}, \mathrm{e}, *}$, Jian Zhou ${ }^{\mathrm{f}}$ \\ ${ }^{a}$ The Logistics Institute-Asia Pacific, National University of Singapore, Singapore \\ ${ }^{b}$ Institute for Risk and Uncertainty, University of Liverpool, Liverpool United Kingdom \\ ${ }^{c}$ Department of Mathematical Sciences, University of Liverpool, Liverpool United Kingdom \\ ${ }^{d}$ Institut fur Bauinformatik, Leibniz Universitat Hannover, Hannover Germany \\ e School of Civil Engineering 85 Shanghai Institute of Disaster Prevention and Relief, Tongji University, China \\ ${ }^{f}$ Department of Management Science and Engineering, Shanghai University, Shanghai China
}

\begin{abstract}
Offshore wind farm is an emerging source of renewable energy, which has been shown to have tremendous potential in recent years. In this blooming area, a key challenge is that the preventive maintenance of offshore turbines should be scheduled reasonably to satisfy the power supply without failure. In this direction, two significant goals should be considered simultaneously as a trade-off. One is to maximise the system reliability and the other is to minimise the maintenance related cost. Thus, a non-linear multi-objective programming model is proposed including two newly defined objectives with thirteen families of constraints suitable for the preventive maintenance of offshore wind farms. In order to solve our model effectively, the nondominated sorting genetic algorithm II, especially for the multi-objective optimisation is utilized and Pareto-optimal solutions of schedules can be obtained to offer adequate support to decision-makers. Finally, an example is given to illustrate the performances of the devised model and algorithm, and explore the relationships of the two targets with the help of a contrast model.
\end{abstract}

Keywords: Reliability, Maintenance, Scheduling, Cost Parameters, Offshore Wind Farms, Multi-objective Programming.

\section{Introduction - Motivation}

The wind energy capacity currently installed in the European Union (EU) can produce 284 TWh of electricity in an average wind year, which is enough to cover $10.2 \%$ of the EU's total electricity consumption $\bigsqcup^{1}$

${ }^{*}$ Corresponding author. Tel.: +49511 7625986.

Email addresses: shuya.zhong@hotmail.com (Shuya Zhong), A.Pantelous@liverpool.ac.uk (Athanasios A. Pantelous), beer@irz.uni-hannover.de (Michael Beer), zhou_jian@shu.edu.cn (Jian Zhou)

${ }^{1}$ The UK remains in Europe with the largest amount of installed offshore wind capacity (45.9\%), followed by Germany (29.9\%), Denmark (11.5\%), Belgium (6.5\%), the Netherlands (3.9\%) and Sweden (1.8\%) (more 
At present, offshore wind power accounts for almost $1.1 \%$ of the EU's total capacity in the electricity consumption. Obviously, offshore wind farms are emerging to be one of the driving sources of energy in the green power world. In the US in May 2014, the U.S. Department of Energy awarded three multi-million demonstration projects planned for the New Jersey, Oregon and Virginia coasts. In theory, the potential benefit and challenge are tremendous [39. In Germany, the ambitious Energiewende (energy transition) programme hopes to generate at least $35 \%$ of its electricity from the green renewable energy by 2020, and by 2050 the share is expected to surpass $80 \%$. Again, offshore wind farms in north coastal parts of Germany play a key role in this direction [42]. Last, but not least, it should also be mentioned the Chinese government is giving considerable weight to exploiting this environmentally friendly resource of energy, particularly along the south-eastern part of its coast line [7].

Maintenance is classified into two main categories: the corrective and the preventive maintenance. The former one is usually performed after a system failure or breakdown while the latter one corresponds to the scheduled actions, which are performed while the system is still operational. Generally speaking, the preventive maintenance (PM) is more beneficial as it may prevent serious losses due to unpredicted failures [32].

This paper is aimed at the PM scheduling of offshore wind farms. For generalised power systems, the primary goal of the PM is to avoid or mitigate failure consequences of the electrical and mechanical parts of the system caused by fatigue cumulative damages and corrosion resistance degradations. PM is able to prevent faults effectively either before they occur or before they develop into major defects. Scheduling means to determine the most satisfied arrangement for the downtime of elements in offshore wind farms that need to be preventively maintained. Hence, our PM scheduling of offshore wind farms is transformed to an interesting optimisation problem, which is useful to different decision-makers in the green energy world.

The rest of the paper is organized as follows. In Section 2, a discussion about the new reliability and economic criteria is provided. Section 3 introduces and reviews the algorithm used for solving our problem. A non-linear multi-objective programming model with thirteen families of constraints for the PM scheduling of offshore wind farms is formulated, as well as its contrast model using the squares of net reserves minimisation objective in Section 4 . Then, the technical parts of Nondominated Sorting Genetic Algorithm II (NSGA-II) are presented in Section 5 and Appendix A. The effectiveness and performance of the proposed and contrast models are illustrated by presenting a numerical example in Section 6, and the results are

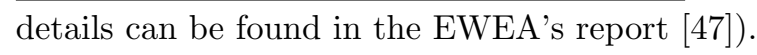


analysed and compared from three main respects.

\section{Objective Functions}

Reliability and economic criteria are the two most popular objectives for the maintenance optimization models of power systems according to the literature to date. However, only a few studies have investigated the maintenance problem particularly designed for the offshore wind energy sector. In the following subsections, an analysis of the two criteria is provided.

\subsection{Reliability Criterion}

In terms of the reliability criterion, there are commonly two mainstream definitions. The first one is related to the required net power reserves to provide the stability in meeting the customer demand, and the second one indicates the deviation of the net power reserves, i.e., the reserve margin. The net power reserve is the balance of the gross reserve after deducting the maintenance loss. For the first type of the reliability measure, Kralj and Petrović [27] suggested that the net reserve generation can be maximised as an optimality criterion. Later, Conejo et al. [6] made a further development and first defined the reliability as the net reserve being divided by the gross reserve. This formulation soon became a classical objective for the maximisation of PM scheduling models. Canto [3] employed it to solve the PM scheduling problem of power plants, and then Canto and Romero [4] extended its application to the problems associated with wind farms integrated power plants.

For the second type of reliability perspective, Egan et al. [16] first proposed that the minimization of the sum of the squares of the reserves (SSR) would prevent the large variations in the net power reserves of each time period, which means the maximization of the reliability. There followed an upsurge in the use of this reliability definition by other scholars, $11,8,10$, 11, 17, 43].

In our paper, we will adjust the first type of the conventional reliability criterion in the PM scheduling of offshore wind farms to model the behavioral attitude of our treatment. As only the customer power demand satisfaction delineated by the power reserve ratio has been studied in the previous definitions from the demand perspective, here the reliability criterion can be better depicted if the decision-maker preferences are also taken into account over a set of choices or attitudes. Moreover, in offshore wind farms, the particularly complex and variable marine environment contributes to the effects of the maintenance and degeneration on the real power reserve which may not have such significant influence on other kinds of power plants [42]. Therefore, another factor, the system sustainability, which means the sustainable 
capability of reserving the power under the combined impacts of the maintenance work and the system degradation in each time period, is of equal importance to be considered in the reliability frame. It can reflect the actually attained power reserve ratio by exponentially adjusting the estimated power reserve ratio. Thus, we propose a novel non-linear definition of the reliability with both of the demand and supply side regards by introducing what we call the "attainment exponent"2, so as to describe the decision-maker's preferences, the power demand satisfaction and the system sustainability simultaneously.

\subsection{Economic Criterion}

With respect to the economic criterion (i.e., the maintenance related cost measure), the minimisation of the cost is always a unified objective definition for almost all maintenance scheduling problems with economic targets. Differences are mainly located in the diverse ingredients of the maintenance cost in different models. The amount of literature in this direction is vast, as many researches have introduced different economic criteria, [12, 23, 25, 31, 40, which have discussed in the offshore wind energy sector. In representative works summarized in Table 11, one can see that there are basically 8 kinds of costs related to the maintenance of power systems: power production, maintenance, start-up, fixed, variable, opportunity, compensation, and failure costs. Specifically, the power production and maintenance costs are the two fundamental costs mostly taken into account when building a cost minimisation objective, and the remaining types of costs are used in different degrees. According to Dahal et al. [9], Ding and Tian [14] and Zhang et al. [52], the market related maintenance cost $\$^{3}$ and the accompanying compensation cost $t^{\text {th }}$ can usually be found in both the preventive and corrective maintenance, while the failure cost (i.e., cost of repair or replacement because of failures) arises only after the breakdown has happened in the mechanical system.

In order to cater to the PM without a power shortage or system failure in this paper, we refer to the definitions of the no-failure maintenance cost presented by Dahal et al. [9] and Dalgic et al. [12] to some degree, including the classical maintenance cost (direct and indirect costs), the start-up cost, the fixed cost, the variable cost and the opportunity cost, owing to opportunity foregone as the economic criterion of our PM scheduling problem of offshore wind farms. Although the power production cost is used in most of the literature, it is not imported in our model because we attribute it to its weak relationship with maintenance works. In

\footnotetext{
${ }^{2}$ This can also be seen a curvature parameter in the reliability index, see Section 4.2.1.

${ }^{3}$ The opportunity cost which partly means the revenue loss due to the power shortage caused by the maintenance outage.

${ }^{4}$ The cost to purchase electricity from other markets to meet customer requirements.
} 


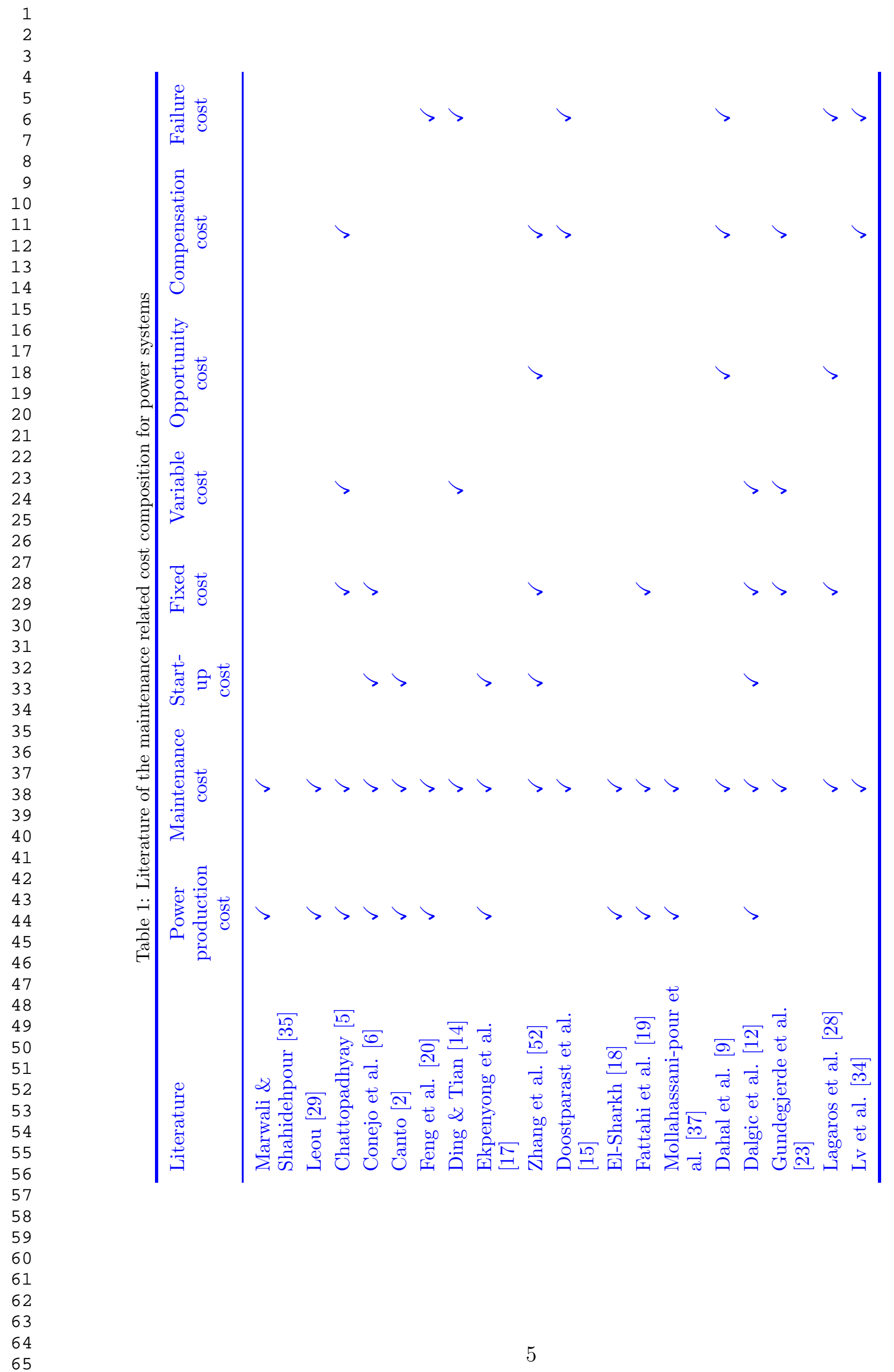


addition, some other cost factors particularly for wind farms are also involved in our definition as indicated by Ding and Tian [14] and Gundegjerde et al. [23], e.g., the fixed cost of sending vessels to wind farms for maintenance, the variable access cost to wind turbines, etc. Thus, a new rational and offshore wind farm-oriented maintenance related cost criterion is well built to conduct an overall weighting.

\section{Optimization Technique}

There are different approaches of multi-objective optimisation for mechanical systems [22, 33. Since reliability and economic criteria are both very important for maintenance scheduling problems of power systems, they should be treated equally to implement a simultaneous optimisation. Actually, models commonly set either reliability maximisation or maintenance cost minimization as their objective functions. Lack of studies on the multi-objective optimisation with classical reliability and cost criteria is a challenge to decision-makers. It is difficult for them to get effective solutions for a reasonable assignment of the two elements in the maintenance scheduling. Therefore, in this paper, for the first time, according to the authors' knowledge, a constrained non-linear multi-objective programming model is constructed for the PM scheduling of offshore wind farms in order to maximise the reliability and minimise the maintenance cost concurrently. Furthermore, for better understanding the performance of the proposed model, we also raise a contrast model, in which the only difference is that the reliability objective is replaced by the SSR minimisation definition. Thus, the relationship between reliability and maintenance cost objectives can be deeply studied by analyzing the trade-offs between the two goals, as well as comparing them using the proposed as well the contrast model.

With respect to the solving methods of the designed multi-objective programming model, the most classical way is to transform it into a single-objective model by the weighted sum approach. As the reliability and maintenance cost objectives with different measures are conflicting with each other, only the sacrificing on one objective can make the other closer to the optimal goal. Thus, this obviously makes the weight setting a process with strong subjectivity and the availability of optimisation results becomes badly affected. Moreover, when such a method is used for seeking multiple satisfying solutions, it has to be applied many times, hopefully finding a different solution at each iteration. If more solutions cannot be obtained, decisionmakers are unable to evaluate each objective by the single solution effectively. In order to overcome the shortcomings, multi-objective evolutionary algorithms (MOEAs) are proposed for their ability to find multiple Pareto-optimal solutions in a single simulation run. The first MOEA, called vector evaluated genetic algorithm (VEGA) was proposed by Schaffer [41. An 
algorithm called nondominated sorting genetic algorithm (NSGA) based on the nondominated

sorting is proposed by Srinivas and Deb [45]. It was later developed by Deb et al. [13] and named NSGA-II, which alleviates high computational complexity of the nondominated sorting, lack of the elitism and use of the sharing parameter.

MOEAs are employed to solve some multi-objective maintenance scheduling models for power systems. Leou [30] put forward a genetic algorithm (GA) combined with the simulated annealing method to solve the unit maintenance scheduling problem with the fitness maximisation objective composed by reliability and cost indices. In the maintenance scheduling optimisation in Yang et al. [50], the Markov model was used to handle reliability and cost objectives, and then in Yang and Chang [49], the same model was rebuilt for energy not served, and operation and expected failure cost objectives. Both models were solved by NSGA-II, so with the imperfect PM maintenance model in Wang and Pham [48. Zhan et al. [51] designed a multi-objective generation maintenance scheduling model, in which five objectives containing the profit maximisation, SSR minimisation and generation cost minimisation were optimized by group search optimizer with multiple producers.

Hence, in our paper, we utilize the NSGA-II, which is able to find a much better spread of solutions and better convergence near the true Pareto-optimal front when compared to other MOEAs, to solve our constrained non-linear multi-objective programming model for the PM scheduling of offshore wind farms. After decision-makers obtain Pareto-optimal solutions from the algorithm, they need to analyse the results and make trade-off decisions for determining an appropriate satisficing solution to support the offshore wind farm project.

\section{Mathematical Model Formulation}

In this section, the formulation of the multi-objective programming model is presented with the objectives of reliability maximization and cost minimisation under several realistic constraints for the PM scheduling problem of offshore wind farms.

\subsection{Notations}

Before we proceed further, indices, parameters and decision variables used in this paper are introduced in Table 2,

\subsection{Mathematical Formulation of Objective Functions}

Our aim is to allocate $m$ turbines in offshore wind farms to implement their maintenance in different time periods, taking into account optimising the system reliability and the maintenance 
Table 2: Notations for the PM scheduling problem of offshore wind farms

\begin{tabular}{|c|c|c|c|}
\hline$m$ & number of turbines in wind farm & $H_{i}$ & helicopter need for maintaining $\mathrm{TR}_{i}$ \\
\hline$i$ & index of offshore wind turbines & $L P_{i}$ & maintenance duration $\mathrm{TR}_{i}$ requires \\
\hline$n$ & number of periods in time horizon & $C^{F^{V}}$ & per unit fixed cost $(€)$ of vessels \\
\hline$t$ & index of time periods & $C^{F^{H}}$ & per unit fixed cost $(€)$ of helicopters \\
\hline $\mathrm{TR}_{i}$ & the $i$ th turbine & $C_{i, t}^{T}$ & total transport cost $(€)$ of $\mathrm{TR}_{i}$ in $\mathrm{PR}_{t}$ \\
\hline $\mathrm{PR}_{t}$ & the $t$ th time period & $C_{i, t}^{A}$ & adjustment cost $(€)$ for $\mathrm{TR}_{i}$ in $\mathrm{PR}_{t}$ \\
\hline$p_{i, t}$ & $\begin{array}{l}\text { power }(\mathrm{MW}) \text { generated by } \mathrm{TR}_{i} \text { in } \\
\mathrm{PR}_{t}\end{array}$ & $C_{i, t}^{C R M}$ & $\begin{array}{l}\text { customer relationship management } \\
\text { cost }(€) \text { for } \mathrm{TR}_{i} \text { in } \mathrm{PR}_{t}\end{array}$ \\
\hline$d_{t}$ & power $(\mathrm{MW})$ required in $\mathrm{PR}_{t}$ & $C_{i, t}$ & $\begin{array}{l}\text { total maintenance cost }(€) \text { for } \mathrm{TR}_{i} \text { in } \\
\mathrm{PR}_{t}\end{array}$ \\
\hline$s_{t}$ & $\begin{array}{l}\text { attainment exponent affecting power } \\
\text { demand satisfaction in } \mathrm{PR}_{t}, s_{t} \geq 0\end{array}$ & $U$ & $\begin{array}{l}\text { time period set not allowed for } \\
\text { maintenance }\end{array}$ \\
\hline$E_{t}$ & gross power reserve $(\mathrm{MW})$ in $\mathrm{PR}_{t}$ & $L T_{t}$ & turbine maintenance capacity in $\mathrm{PR}_{t}$ \\
\hline$e_{t}$ & net power reserve $(\mathrm{MW})$ in $\mathrm{PR}_{t}$ & $L_{i}$ & maintenance deadline of $\mathrm{TR}_{i}\left(\mathrm{PR}_{L_{i}}\right)$ \\
\hline$r_{t}$ & reliability $(\%)$ in $\mathrm{PR}_{t}$ & $A M_{t}$ & number of available manpower in $\mathrm{PR}_{t}$ \\
\hline$R$ & system reliability (\%) of wind farm & $A V_{t}$ & number of available vessels in $\mathrm{PR}_{t}$ \\
\hline$C_{t}^{M^{V}}$ & vessel manpower cost $(€)$ in $\mathrm{PR}_{t}$ & $A H_{t}$ & number of available helicopters in $\mathrm{PR}_{t}$ \\
\hline$C_{t}^{M^{H}}$ & helicopter manpower cost $(€)$ in $\mathrm{PR}_{t}$ & $z_{i}$ & distance $(\mathrm{km})$ from shore to $\mathrm{TR}_{i}$ \\
\hline$C_{t}^{M^{L}}$ & onshore manpower cost $(€)$ in $\mathrm{PR}_{t}$ & $q^{V}$ & vessel gas emission $(\mathrm{kg} / \mathrm{kg} \cdot \mathrm{km})$ \\
\hline$M_{i}^{V}$ & vessel manpower demand for $\mathrm{TR}_{i}$ & $q^{H}$ & helicopter gas emission $(\mathrm{kg} / \mathrm{kg} \cdot \mathrm{km})$ \\
\hline$M_{i}^{H}$ & helicopter manpower demand for $\mathrm{TR}_{i}$ & $\bar{w}$ & average weight of an employee $(\mathrm{kg})$ \\
\hline$M_{i}^{L}$ & onshore manpower demand for $\mathrm{TR}_{i}$ & $E Q_{i}^{V}$ & equipment $(\mathrm{kg})$ on vessels for $\mathrm{TR}_{i}$ \\
\hline$C_{i, t}^{M}$ & $\begin{array}{l}\text { total manpower cost }(€) \text { for } \mathrm{TR}_{i} \text { in } \\
\mathrm{PR}_{t}\end{array}$ & $E Q_{i}^{H}$ & equipment $(\mathrm{kg})$ on helicopters for $\mathrm{TR}_{i}$ \\
\hline$C_{i, t}^{E Q}$ & equipment cost $(€)$ for $\mathrm{TR}_{i}$ in $\mathrm{PR}_{t}$ & $L V_{t}$ & permitted moving vessels in $\mathrm{PR}_{t}$ \\
\hline$C_{i, t}^{I}$ & infrastructure cost $(€)$ for $\mathrm{TR}_{i}$ in $\mathrm{PR}_{t}$ & $L H_{t}$ & permitted moving helicopters in $\mathrm{PR}_{t}$ \\
\hline$C_{i, t}^{E M}$ & $\begin{array}{l}\text { environmental monitoring cost }(€) \\
\text { for } \mathrm{TR}_{i} \text { in } \mathrm{PR}_{t}\end{array}$ & $G H G$ & $\begin{array}{l}\text { greenhouse gas emission standard } \\
\text { regulated by the industry }(\mathrm{kg})\end{array}$ \\
\hline$C_{i, t}^{S^{V}}$ & $\begin{array}{l}\text { unit vessel transport cost }(€) \text { for } \mathrm{TR}_{i} \\
\text { in } \mathrm{PR}_{t}\end{array}$ & $x_{i, t}$ & $\begin{array}{l}0-1 \text { decision variable denoting the } \\
\text { maintenance status of } \mathrm{TR}_{i} \text { in } \mathrm{PR}_{t}\end{array}$ \\
\hline$C_{i, t}^{S^{H}}$ & $\begin{array}{l}\text { unit helicopter transport cost }(€) \text { for } \\
\mathrm{TR}_{i} \text { in } \mathrm{PR}_{t}\end{array}$ & $b_{i, t}$ & $\begin{array}{l}0-1 \text { decision variable denoting the } \\
\text { starting state of } \mathrm{TR}_{i} \text { in } \mathrm{PR}_{t}\end{array}$ \\
\hline$V_{i}$ & vessel demand for maintaining $\mathrm{TR}_{i}$ & & \\
\hline
\end{tabular}


cost simultaneously. Since the two goals are contradicting, satisfying results can be derived only after recommending appropriate trade-off decision-making strategies.

\subsubsection{System Reliability Maximization Objective}

The first objective function is to maximise the system reliability. The reliability of the whole offshore wind farm system means the customer demand satisfaction for enough electricity is reserved, and simultaneously to consider the effects of the sustainability.

In our problem, dual influences to the system reliability which are brought by maintenance are taken into consideration. On the one hand, there should always be sufficient power generated for normal market consumption and inevitably for satisfying on-peak demand while some turbines stop working due to maintenance. Therefore, performing the necessary maintenance makes the energy generation decrease, resulting in increasing the probability that the power demand cannot be fully satisfied. On the other hand, the maintenance can fight against corrosion and the degradation of the substructures of turbines, and attempts to reduce the risk of serious grid breakdowns. Thereby, three different possible effects emerge to provide actual achievements of the customer power demand satisfaction, i.e., the power reserve ratio. One is that the service life of turbines is extended and the sustainable development of the system is promoted, another is that the system maintains balance to guarantee the average level, and the third is that the system is still getting worse after maintenance because of some deep-rooted or irreversible degenerations.

The system reliability $R$ is the average of reliabilities $r_{t}$ in all periods, which are defined as exponentials of the attainment factor $s_{4}{ }^{5}$ with the base measuring the proportion of the net power reserve $e_{t}$ to the gross power reserve $E_{t}$. Thus, the reliability $r_{t}$ in $\mathrm{PR}_{t}$ is

$$
r_{t}=\left(e_{t} / E_{t}\right)^{s_{t}},
$$

in which the gross power reserve $E_{t}(\mathrm{MW})$ means to deduct the customer electricity demand from the amount generated by all turbines, i.e.,

$$
E_{t}=\sum_{i=1}^{m} p_{i, t}-d_{t}
$$

and the net power reserve $e_{t}$ (MW) also needs to subtract the shutdown loss of the energy production caused by the maintenance as

\footnotetext{
${ }^{5}$ Actually, in this paper, we recommend for the very first time according to the authors' knowledge, the use of an isoelastic function (or in another word, the use of a power function) to model the behavioral attitude of our treatment, see Section 2.1. The isoelastic utility function is a special case of the hyperbolic absolute risk aversion (HARA) utility functions, and is used in analyses either including or not including the underlying risk. For more details, see 44 among numerous others.
} 


$$
e_{t}=\sum_{i=1}^{m} p_{i, t}\left(1-x_{i, t}\right)-d_{t}, \text { where } x_{i, t} \in\{0,1\} \text {. }
$$

So the equivalent form of the reliability $r_{t}$ in Eq. (1) is

$$
r_{t}=\left[\frac{\sum_{i=1}^{m} p_{i, t}\left(1-x_{i, t}\right)-d_{t}}{\sum_{i=1}^{m} p_{i, t}-d_{t}}\right]^{s_{t}} .
$$

It can be seen that the value of the power reserve ratio $e_{t} / E_{t}$ partly reflects whether the system is reliable in $\mathrm{PR}_{t}$. It is also noted the lower bound is that the power reserve should at least be enough to satisfy the customer requirement though some turbines stop working for maintenance, i.e., $e_{t}=0, e_{t} / E_{t}=0, r_{t}=0$, and the upper bound is that the net power reserve equals to the gross power reserve when there is no turbine in maintenance in $\mathrm{PR}_{t}$, i.e., $e_{t}=1, e_{t} / E_{t}=1, r_{t}=1$

Regarding the exponent, i.e., the attainment factor $s_{t}$, since the base is $e_{t} / E_{t} \in[0,1]$, the reliability $r_{t}$ decreases from 1 approaching to 0 with $s_{t} \in[0,+\infty)$ increasing according to properties of the exponential function. It also gives the power reserve ratio $e_{t} / E_{t}$ three different kinds of effects by different parameter values as follows:

(1) "Positive" effect: $r_{t}=\left(e_{t} / E_{t}\right)^{s_{t}}>e_{t} / E_{t}$, when $s_{t} \in[0,1)$. The reliability index is upgraded by the decision-maker.

(2) "Neutral" effect: $r_{t}=\left(e_{t} / E_{t}\right)^{s_{t}}=e_{t} / E_{t}$, when $s_{t}=1$ : This means that impact of the decision-maker is the same. There is neither an upgrade nor a downgrade of the reliability index.

(3) "Negative" effect: $r_{t}=\left(e_{t} / E_{t}\right)^{s_{t}}<e_{t} / E_{t}$, when $s_{t} \in(1,+\infty)$ : In this case, there is a downgrade.

Especially, for purpose of better understanding the positive, neutral and negative effects brought by different attainment exponents $s_{t}$, Fig. 1 provides an illustrative example of $r_{t}=$ $(2 / 5)^{s_{t}}$, in which each point stands for a type of effect, respectively.

Since the electricity generated and demanded in each time period is an approximate estimate in terms of the historical data, the power reserve ratio $e_{t} / E_{t}$ which has eliminated the influence of the maintenance downtime, as well as the attainment exponent $s_{t}$ reflecting the effects of decision-makers attitude, can coordinate to represent the actual achievement of the customer power demand satisfaction. The power reserve ratio and the attainment exponent are two constitutive elements of the reliability $r_{t}$ in $\mathrm{PR}_{t}$. Then the system reliability $R$ can be defined by averaging individual reliabilities $r_{t}$ as

$$
R=\sum_{t=1}^{n} \frac{1}{n} r_{t}
$$




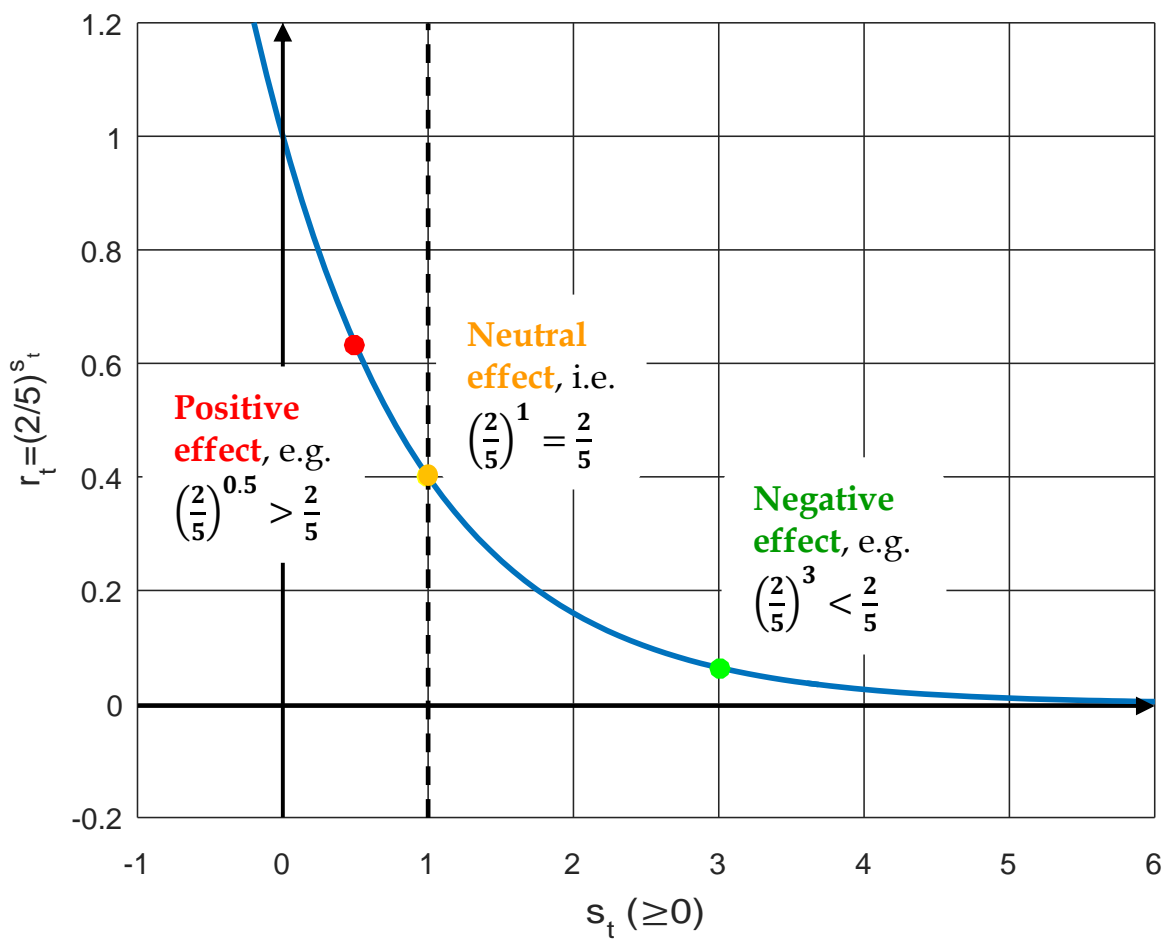

Fig. 1: An example for three types of effects of attainment exponents with $r_{t}=(2 / 5)^{s_{t}}$ and $s_{t}=0.5,1$ and 3 , respectively.

in which the weight coefficient $1 / n$ of $r_{t}$ are for normalization to adjust $R$ into the range $[0,1]$. According to Eqs. (1) and (4), the system reliability $R$ is equivalent to

$$
R=\sum_{t=1}^{n} \frac{1}{n}\left(\frac{e_{t}}{E_{t}}\right)^{s_{t}}=\sum_{t=1}^{n} \frac{1}{n}\left[\frac{\sum_{i=1}^{m} p_{i, t}\left(1-x_{i, t}\right)-d_{t}}{\sum_{i=1}^{m} p_{i, t}-d_{t}}\right]^{s_{t}} .
$$

Notably, due to $n$ reliabilities $r_{t}$ constituting the final reliability $R$, it means that there are $n$ attainment exponents $s_{t}$ needed to be settled based on three different types of effects. As it is difficult to collect the exact data of the effects due to the unknown degradation status and the maintenance capability especially for newly grid-connected offshore wind farms, a feasible scheme is to draw support from the decision-maker's experience. Over the entire time horizon, decision-maker's attitudes and preferences to the maintenance versus degradation trend of the offshore wind power project. Thus, in what follows, we test some predefined behavioral attitudes of the decision-makers. Obviously, the proposed four categories, "fully rational", "optimism biased", "wait-and-see attitudes" and "pessimism biased" are initiating and inspiring, rather than exhaustive and conclusive for the research on maintenance, and more generally speaking, in the behavioral approach of the reliability index and our multi-objective constrained optimization problem. So, let us define the four categories of attitudes:

(1) When decision-makers are fully rational, and all the three effects appear in sequence over the time. Specifically, they believe that if turbines are maintained as much as possible in the 
early stage of all $n$ periods, attainment exponents $s_{t}$ give the customer demand satisfaction positive impacts because it is not only easier to solve the degradation but also benefits the system survivability for the duration. When it comes to the mid-term stage, the effects of $s_{t}$ tend to be neutral as the system performance gradually weakens. Along with the continuous decline, no matter that the turbines have already been maintained before the latter stage or are precisely in maintenance, the advantages from the maintenance are overtaken by cumulative damages and failure risks. Accordingly, negative influences of $s_{t}$ on customer satisfaction occur in the latter stage. Therefore, attainment exponents $s_{1}, s_{2}, \ldots, s_{n}$ are selected from the three sets $[0,1),\{1\},(1,+\infty)$.

(2) When decision-makers are optimism biased, they are always inclined to think that a higher real customer demand satisfaction on positive effects of attainment exponents $s_{t}$ can be reached. It means that the maintenance is able to overwhelm the deterioration over all $n$ periods and the system reliability remains at a high level. Therefore, when decision-makers have such a preference, all attainment exponents $s_{1}, s_{2}, \ldots, s_{n}$ are chosen from the interval $[0,1)$.

(3) When decision-makers take wait-and-see attitudes, which refer to no clear or specific preference firmly in mind, they think that efforts of the maintenance and the deterioration can be perceived as merits equal demerits. No bias on the real achieved customer satisfaction and reliability happens in any period over the time horizon. Thus, all attainment exponents $s_{1}, s_{2}, \ldots, s_{n}$ equal to 1 , i.e., no exponents when decision-makers are conservative, which suggests that it transforms to the first conventional reliability criterion of the PM scheduling (the power reserve ratio). Therefore, the classical power reserve ratio is included as one of the particular scenarios in our reliability formulation, so that the limitation of the original reliability design is reflected and improved.

(4) When decision-makers are pessimism biased, it is thought that negative effects of attainment exponents $s_{t}$ take up whole time periods owing to all kinds of degradations and risks in the severe marine environment, even the maintenance is essentially not powerful enough to improve the instability of the wind farm system. Consequently, all attainment exponents $s_{1}, s_{2}, \ldots, s_{n}$ can be picked from the interval $(1,+\infty)$.

In accordance with the four kinds of decision-maker's attitudes, the system reliability $R$ can be determined explicitly by the weighted sum of reliabilities $r_{t}$. Hence, the first non-linear objective function of our model is the system reliability maximisation:

$$
\max R=\max _{\boldsymbol{X}} \sum_{t=1}^{n} \frac{1}{n}\left[\frac{\sum_{i=1}^{m} p_{i, t}\left(1-x_{i, t}\right)-d_{t}}{\sum_{i=1}^{m} p_{i, t}-d_{t}}\right]^{s_{t}} .
$$




\subsubsection{Maintenance Cost Minimization Objective}

The second objective function is to minimize the maintenance related cost. In the following, the seven costs including the manpower, equipment, infrastructure, environmental monitoring, transportation, adjustment and customer relationship management costs are introduced explicitly:

(1) Manpower cost $C_{i, t}^{M}$ : the direct maintenance cost for technical and administrative labour in maintaining offshore wind farms, and the indirect maintenance cost for staff welfare. It can be expressed as

$$
C_{i, t}^{M}=C_{t}^{M^{V}} M_{i}^{V}+C_{t}^{M^{H}} M_{i}^{H}+C_{t}^{M^{L}} M_{i}^{L},
$$

where $C_{t}^{M^{V}}, C_{t}^{M^{H}}$, and $C_{t}^{M^{L}}$ are per capita manpower costs in $\mathrm{PR}_{t}$ for employees working on vessels, helicopters and land, and $M_{i}{ }^{V}, M_{i}{ }^{H}$, and $M_{i}{ }^{L}$ are corresponding amounts of manpower needed for maintaining $\mathrm{TR}_{i}$.

(2) Equipment $\operatorname{cost} C_{i, t}^{E Q}$ : the direct maintenance cost for purchasing spare parts, material and equipment required for the maintenance of $\mathrm{TR}_{i}$ in $\mathrm{PR}_{t}$, as well as the indirect maintenance cost for equipment storage and testing.

(3) Infrastructure cost $C_{i, t}^{I}$ : the start-up cost of enabling infrastructures (i.e., ports, docks, helipads, etc.) that support the maintenance of $\mathrm{TR}_{i}$ in $\mathrm{PR}_{t}$, and the indirect maintenance cost of operating and maintaining them.

(4) Environmental monitoring cost $C_{i, t}^{E M}$ : the indirect maintenance cost of monitoring whether the maintenance activities seriously influence the marine environment around offshore wind farms beyond acceptable thresholds, i.e., the air and livings of marine creatures and bird species. Meanwhile, considering the complexity of the marine environment, dynamic monitoring is also essential for real-time weather forecasts on the sea, in order to judge whether it is appropriate for implementing the offshore maintenance of $\mathrm{TR}_{i}$ in $\mathrm{PR}_{t}$.

(5) Transportation cost $C_{i, t}^{T}$ : the fixed cost of employing and maintaining vessels and helicopters, and the variable cost of marine and air shipments to offshore wind turbines, including fuel cost and the cost of remaining at turbines for supporting maintenance activities. As to the maintenance of offshore wind farms, costs related to the manpower and equipment transportation account for a large proportion of the total maintenance related cost because of the special environment of the sea. It is formulated as

$$
C_{i, t}^{T}=\left(C^{F^{V}} V_{i}+C^{F^{H}} H_{i}\right) / L P_{i}+\left(C_{i, t}^{S^{V}} V_{i}+C_{i, t}^{S^{H}} H_{i}\right) .
$$

The first term means the fixed cost for the use of vehicles, in which $C^{F^{V}}$ and $C^{F^{H}}$ are per unit fixed costs of vessels and helicopters when putting them into use, and $V_{i}$ and $H_{i}$ are the 
respective quantities of two vehicles the maintenance of $\mathrm{TR}_{i}$ requires. Since this cost is incurred once when starting using a vessel or helicopter, it is divided by $L P_{i}$, which is the maintenance duration time of $\mathrm{TR}_{i}$. In the second term, $C_{i, t}^{S^{V}}$ and $C_{i, t}^{S^{H}}$ are average variable shipment costs per vessel and helicopter, along with their fuel costs and waiting costs for maintaining $\mathrm{TR}_{i}$ in $\mathrm{PR}_{t}$.

(6) Adjustment $\operatorname{cost} C_{i, t}^{A}$ : the opportunity cost for adjusting the maintenance when the schedule needs to be altered because of changes in weather and power demand and some other emergency situations. As the maintenance is scheduled according to estimated data, some adjustments are required for the deployment of the maintenance. Thus the adjustment cost for $\mathrm{TR}_{i}$ in $\mathrm{PR}_{t}$ arises.

(7) Customer relationship management (CRM) cost $C_{i, t}^{C R M}$ : the opportunity cost for maintaining the customer relationship. Although the maintenance aims at enhancing the system reliability of offshore wind farms, the risk of power shortage may increase due to the maintenance downtime. In order to retain customer satisfaction and loyalty, the CRM cost for $\mathrm{TR}_{i}$ in $\mathrm{PR}_{t}$ is invested to analyse customers, promote the benefits of the renewable wind energy, and make more long-term potential contracts possible.

Thus, the above seven elements constitute the total maintenance cost $C_{i, t}$ of $\mathrm{TR}_{i}$ in $\mathrm{PR}_{t}$ as

$$
C_{i, t}=C_{i, t}^{M}+C_{i, t}^{E Q}+C_{i, t}^{I}+C_{i, t}^{E M}+C_{i, t}^{T}+C_{i, t}^{A}+C_{i, t}^{C R M}
$$

where each item stands for one ingredient of the PM cost for offshore wind farms. Thereby, the maintenance cost minimization objective function of our problem can be presented as

$$
\min _{\boldsymbol{X}} \sum_{i=1}^{m} \sum_{t=1}^{n} C_{i, t} x_{i, t}=\min _{\boldsymbol{X}} \sum_{i=1}^{m} \sum_{t=1}^{n}\left(C_{i, t}^{M}+C_{i, t}^{E Q}+C_{i, t}^{I}+C_{i, t}^{E M}+C_{i, t}^{T}+C_{i, t}^{A}+C_{i, t}^{C R M}\right) x_{i, t},
$$

in which the manpower cost $C_{i, t}^{M}$ and the transportation $\operatorname{cost} C_{i, t}^{T}$ are detailed by their definitions, respectively, see Eqs. (8) and (9).

Notably, the environmental monitoring cost $C_{i, t}^{E M}$ and the transportation cost $C_{i, t}^{T}$ are designed especially for the PM of offshore wind farms due to the specificity of the marine environment, while the other five costs can also apply to that of general power systems.

\subsection{Constraints}

The constraints should not only be well applicable for the PM scheduling problem of general power plants, but also carefully devised for that of offshore wind farms. In total, thirteen families of constraints are proposed: supply and demand, maintenance necessity, maintenance continuity, duration, period, priority, and deadline constraints are the basic ones for the PM 
scheduling problem of power systems, see [1, 4. However, weather, manpower, vehicle, greenhouse gas emission, marine ecosystem, and bird population constraints are proposed by Dalgic et al. [12], Gundegjerde et al. [23], Hassan [24], Karyotakis [26] and Michler-Cieluch et al. [36], and particularly designed for offshore wind power systems coping with the harsh offshore environment.

\subsubsection{Supply and demand constraints}

The electric power virtually generated which has taken out the maintenance downtime loss should be able to cover the customer demand entirely. So the supply and demand constraints guarantee that the power shortage never occurs in any time period,

$$
\sum_{i=1}^{m} p_{i, t}\left(1-x_{i, t}\right)-d_{t} \geq 0, \quad t=1,2, \ldots, n,
$$

which are namely to restrict net power reserves $e_{t}(\mathrm{MW})$ in Eq. (3) no less than 0.

\subsubsection{Maintenance necessity constraints}

The maintenance of wind turbines that are especially located offshore costs enormous manpower and material resources, so every turbine is set to be maintained only once over the time horizon without any pause halfway,

$$
\sum_{t=1}^{n} b_{i, t}=1, \quad i=1,2, \ldots, m
$$

This means for any $\mathrm{TR}_{i}$, it needs to be maintained once and for all during all $n$ time periods.

\subsubsection{Maintenance continuity constraints}

When $\mathrm{TR}_{i}$ starts to be maintained, it enters the downtime and maintenance works cannot be stopped before they are all finished. The maintenance continuity constraints clarify the relationships between the two sets of decision variables $x_{i, t}$ and $b_{i, t}$. The decision variables meet the following logical relationships

$$
x_{i, t} \geq b_{i, t}, \quad i=1,2, \ldots, m, t=1,2, \ldots, n,
$$

which imply that when $b_{i, t}=1, x_{i, t}=1$ must hold. It means that when $\mathrm{TR}_{i}$ begins maintenance at the beginning of $\mathrm{PR}_{t}$, it must be in maintenance during the whole period. Moreover, Eq. (14) shows that when $b_{i, t}=0, x_{i, t}=0$ or 1 , i.e., if the maintenance of $\mathrm{TR}_{i}$ does not start at $\mathrm{PR}_{t}$, it may not or may still be in maintenance in this period. Besides, another two additional relationships are derived as follows, 


$$
\begin{aligned}
& x_{i, t}-x_{i, t-1} \leq b_{i, t}, \quad i=1,2, \ldots, m, t=1,2, \ldots, n \\
& x_{i, t}+x_{i, t-1}+b_{i, t}<3, \quad i=1,2, \ldots, m, t=1,2, \ldots, n
\end{aligned}
$$

where $x_{i, t-1}=0$ when $t=1$. They limit the relationships of maintenance activities in two successive time periods $\mathrm{PR}_{t-1}$ and $\mathrm{PR}_{t}$.

\subsubsection{Duration constraints}

As to $\mathrm{TR}_{i}$, the duration of periods for its maintenance is predetermined and fixed by the project. The maintenance duration constraints limit are

$$
\sum_{t=1}^{n} x_{i, t}=L P_{i}, \quad i=1,2, \ldots, m
$$

where $L P_{i}$ is the number of time periods that $\mathrm{TR}_{i}$ needs for maintenance.

\subsubsection{Period constraints}

In any $\mathrm{PR}_{t}$, the power generation needs to satisfy the demand market. As turbines in maintenance stop working and have no electricity to output, the total number of turbines in maintenance in $\mathrm{PR}_{t}$ should be restricted to an upper limit.

$$
\sum_{i=1}^{m} x_{i, t} \leq L T_{t}, \quad t=1,2, \ldots, n
$$

where $L T_{t}$ is the presupposed limit of turbines shut down in $\mathrm{PR}_{t}$.

\subsubsection{Priority constraints}

Sometimes the maintenance of a single turbine needs to be fully done before another due to a variety of reasons, so the priority constraints set the precedence of the maintenance for two different turbines over the time horizon. We assume that the maintenance of $\mathrm{TR}_{i}$ is prior to that of $\mathrm{TR}_{j}$, then

$$
\sum_{k=1}^{t} b_{i, k}-b_{j, t} \geq 0, \quad i=1,2, \ldots, m, j \neq i, t=1,2, \ldots, n,
$$

where $k$ represents the index of time periods from $\mathrm{TR}_{1}$ to $\mathrm{TR}_{t}$, and

$$
x_{i, t}+x_{j, t} \leq 1, \quad i=1,2, \ldots, m, j \neq i, t=1,2, \ldots, n .
$$

It can be seen that the whole maintenance duration of $\mathrm{TR}_{i}$ should remain ahead of that of $\mathrm{TR}_{j}$, and there is not any overlap period between the maintenance of the two turbines.

\subsubsection{Deadline constraints}

In some cases, the maintenance of a turbine has a deadline. If the maintenance of $\mathrm{TR}_{i}$ is stated to be accomplished by the end of $\mathrm{PR}_{L_{i}}$, there is a deadline constraint to compel $\mathrm{TR}_{i}$ to start maintaining no later than $\mathrm{PR}_{L_{i}-L P_{i}+1}$ as, 


$$
\sum_{t=1}^{L_{i}-L P_{i}+1} b_{i, t}=1, \quad i=1,2, \ldots, m .
$$

Thus, $\mathrm{TR}_{i}$ would have enough time to finish the maintenance before its deadline.

\subsubsection{Weather constraints}

The weather constraints are particular to the natural marine environment that only offshore wind energy confronts. Considering the complex and volatile weather conditions such as wind speed, wave height, flight visibility, marine storm, etc., the maintenance of offshore wind farms cannot be implemented in some periods [39]. For instance, the wind in winter is usually stronger than in other seasons, so the use of vessels, helicopters and crews are unsafe for use for maintenance in winter. Additionally, the high wind speed results in the rise of energy production and the customer electricity demand also increases considerably during the winter season. These weather factors encourage decision-makers to arrange maintenance in winter as little as possible. The weather constraints which restrict the maintenance execution are formulated as follows,

$$
\sum_{t \in U} x_{i, t}=0, \quad i=1,2, \ldots, m,
$$

where $U$ is the set of periods not permitted for maintenance due to the weather effect on the sea.

\subsubsection{Manpower constraints}

In any period $t$, crew numbers related to the maintenance should be guaranteed. Manpower, both for maintenance activities to offshore wind turbines by vessels and helicopters and for remote monitoring, control and logistics onshore cannot exceed the total available number of employees in $\mathrm{PR}_{t}$. Thus, the manpower constraints are expressed as

$$
\sum_{i=1}^{m}\left(M_{i}{ }^{V}+M_{i}{ }^{H}+M_{i}{ }^{L}\right) x_{i, t} \leq A M_{t}, \quad t=1,2, \ldots, n,
$$

where $M_{i}{ }^{V}, M_{i}{ }^{H}$, and $M_{i}{ }^{L}$, respectively stand for all technical and administrative manpower required on vessels and helicopters and on land for maintaining $\mathrm{TR}_{i}$, and $A M_{t}$ is the total number of idle employees in $\mathrm{PR}_{t}$.

\subsubsection{Vehicle constraints}

Vessels and helicopters are vehicles for transiting crews and equipment from shore side to offshore turbines to operate maintenance works. The vehicle constraints restrict the numbers of vessels and helicopters used for maintenance in $\mathrm{PR}_{t}$, which cannot exceed the total available number of vehicles in that period. Similar to the forms of the above manpower constraints, the vehicle constraints can be presented separately for vessels and helicopters as 


$$
\begin{aligned}
& \sum_{i=1}^{m} V_{i} x_{i, t} \leq A V_{t}, \quad t=1,2, \ldots, n, \\
& \sum_{i=1}^{m} H_{i} x_{i, t} \leq A H_{t}, \quad t=1,2, \ldots, n,
\end{aligned}
$$

where $V_{i}$ and $H_{i}$ are numbers of vessels and helicopters $\mathrm{TR}_{i}$ requires, respectively to transport manpower and equipment for offshore maintenance according to different turbine locations, and $A V_{t}$ and $A H_{t}$ are the corresponding unoccupied vehicle numbers in $\mathrm{PR}_{t}$.

\subsubsection{Greenhouse gas emission constraints}

Vessels and helicopters used to transfer crews and equipment for offshore maintenance are supplied with fossil fuel, and then discharge various greenhouse gases mainly including carbon dioxide $\left(\mathrm{CO}_{2}\right)$, methane $\left(\mathrm{CH}_{4}\right)$, nitrous oxide $\left(\mathrm{N}_{2} \mathrm{O}\right)$, hydrofluorocarbons (HFCs), perfluorocarbons ( $\mathrm{PFCs})$, and sulphur hexafluoride $\left(\mathrm{SF}_{6}\right)$. These gases pollute the atmosphere, and cause greenhouse effect and global warming as well. Therefore, greenhouse gas emissions $(\mathrm{g} / \mathrm{km})$ in the maintenance system should also strictly comply with national emission standards. Thus, to be an environmentally friendly offshore wind energy project, the total gas emission mass of transfer vessels and helicopters in any period over the maintenance time horizon can be no more than the industrial emission standard as follow,

$$
\sum_{i=1}^{m} 2 z_{i} b_{i, t}\left[q^{V}\left(\bar{w} M_{i}^{V}+E Q_{i}^{V}\right)+q^{H}\left(\bar{w} M_{i}^{H}+E Q_{i}^{H}\right)\right] \leq G H G, \quad t=1,2, \ldots, n,
$$

where $z_{i}$ is the distance $(\mathrm{km})$ from the docking point onshore to $\mathrm{TR}_{i}$ offshore, $q^{V}$ and $q^{H}$ $(\mathrm{kg} / \mathrm{kg} \cdot \mathrm{km})$ are respective kilograms of greenhouse gases that vessels and helicopters emit per kilogram weight of the items they bear for transport per kilometre, $M_{i}{ }^{V}$ and $M_{i}{ }^{H}$, respectively represent the number of manpower required on vessels and helicopters for maintaining $\mathrm{TR}_{i}$, $\bar{w}$ means the average weight $(\mathrm{kg})$ of an employee, $E Q_{i}^{V}$ and $E Q_{i}^{H}$ indicate the weight $(\mathrm{kg})$ of the equipment carried by vessels and helicopters, respectively for the maintenance of $\mathrm{TR}_{i}$, and $G H G$ is the emission standard $(\mathrm{kg})$ regulated by the industry.

\subsubsection{Marine ecosystem constraints}

Apart from the atmospheric pollution transport vehicles for maintenance of offshore wind farms bring about, they also make contributions to the ecosystem. Fleets of vessels, the primary vehicles navigating on the sea for maintenance activities disturb the living environment of marine species to some extent. For example, the fuel leakage and marine litter from vessels shuttling back and forth can damage the living environment of marine life. The movement and noise they make scare the fish school, and can have negative effects on fish migration and also influence the mariculture. In order to protect the marine ecosystem from impacts of moving 
vessels, the number of navigating vessels in $\mathrm{PR}_{t}$ should be no more than the ceiling stipulated by the project based on actual marine situations as

$$
\sum_{i=1}^{m} V_{i}\left(b_{i, t}+b_{i, t-L P_{i}+1}\right) \leq L V_{t}, \quad t=1,2, \ldots, n,
$$

where $V_{i}$ is the amount of vessels to transit for $\mathrm{TR}_{i}, b_{i, t}$ and $b_{i, t-L P_{i}+1}$ are respective indications of vehicles out and return journeys because of offshore maintenance in $\mathrm{PR}_{t}$, and $L V_{t}$ is the total permitted amount of moving vessels in each period.

\subsubsection{Bird population constraints}

Low-flying helicopters on out and return journeys for offshore maintenance impact the life and migration of the bird population. Since birds are sensitive to human disturbance, special care is required when using helicopters to transit crews and equipment for maintenance, in order to avoid causing difficulties for birds or endangering their lives. Hence, the number of navigating helicopters in each period should be tightly controlled for bird population protection. It cannot exceed the upper limit $L H_{t}$ in $\mathrm{PR}_{t}$ as

$$
\sum_{i=1}^{m} H_{i}\left(b_{i, t}+b_{i, t-L P_{i}+1}\right) \leq L H_{t}, \quad t=1,2, \ldots, n,
$$

where $H_{i}$ is the helicopter quantity for transportation while maintaining $\mathrm{TR}_{i}$, and the bird population constraints have similar formulations to the marine ecosystem constraints proposed above.

\subsection{Multi-objective Programming Model and Contrast Model}

In terms of the above two objective functions and thirteen constraints, a non-linear multiobjective programming model for our maintenance scheduling optimisation problem of offshore wind farms is proposed as follows,

$$
\left\{\begin{array}{l}
\max _{\boldsymbol{X}} \sum_{t=1}^{n} \frac{1}{n}\left[\frac{\sum_{i=1}^{m} p_{i, t}\left(1-x_{i, t}\right)-d_{t}}{\sum_{i=1}^{m} p_{i, t}-d_{t}}\right]^{s t} \\
\min _{\boldsymbol{X}} \sum_{i=1}^{m} \sum_{t=1}^{n}\left(C_{i, t}^{M}+C_{i, t}^{E Q}+C_{i, t}^{I}+C_{i, t}^{E M}+C_{i, t}^{T}+C_{i, t}^{A}+C_{i, t}^{C R M}\right) x_{i, t}
\end{array}\right.
$$


subject to:

$$
\begin{aligned}
& \sum_{i=1}^{m} p_{i, t}\left(1-x_{i, t}\right)-d_{t} \geq 0, \quad t=1,2, \ldots, n \\
& \sum_{t=1}^{n} b_{i, t}=1, \quad i=1,2, \ldots, m \\
& x_{i, t} \geq b_{i, t}, \quad i=1,2, \ldots, m, t=1,2, \ldots, n \\
& x_{i, t}-x_{i, t-1} \leq b_{i, t}, \quad i=1,2, \ldots, m, t=1,2, \ldots, n \\
& x_{i, t}+x_{i, t-1}+b_{i, t}<3, \quad i=1,2, \ldots, m, t=1,2, \ldots, n \\
& \sum_{t=1}^{n} x_{i, t}=L P_{i}, \quad i=1,2, \ldots, m \\
& \sum_{i=1}^{m} x_{i, t} \leq L T_{t}, \quad t=1,2, \ldots, n \\
& \sum_{k=1}^{t} b_{i, k}-b_{j, t} \geq 0, \quad i=1,2, \ldots, m, j \neq i, t=1,2, \ldots, n \\
& x_{i, t}+x_{j, t} \leq 1, \quad i=1,2, \ldots, m, j \neq i, t=1,2, \ldots, n \\
& \sum_{t=1}^{L_{i}-L P_{i}+1} b_{i, t}=1, \quad i=1,2, \ldots, m \\
& \sum_{t \in U} x_{i, t}=0, \quad i=1,2, \ldots, m \\
& \sum_{i=1}^{m}\left(M_{i}{ }^{V}+M_{i}^{H}+M_{i}{ }^{L}\right) x_{i, t} \leq A M_{t}, \quad t=1,2, \ldots, n \\
& \sum_{i=1}^{m} V_{i} x_{i, t} \leq A V_{t}, \quad t=1,2, \ldots, n \\
& \sum_{i=1}^{m} H_{i} x_{i, t} \leq A H_{t}, \quad t=1,2, \ldots, n \\
& \sum_{i=1}^{m} 2 z_{i} b_{i, t}\left[q^{V}\left(\bar{w} M_{i}^{V}+E Q_{i}^{V}\right)+q^{H}\left(\bar{w} M_{i}^{H}+E Q_{i}^{H}\right)\right] \leq G H G, \quad t=1,2, \ldots, n \\
& \sum_{i=1}^{m} V_{i}\left(b_{i, t}+b_{i, t-L P_{i}+1}\right) \leq L V_{t}, \quad t=1,2, \ldots, n \\
& \sum_{i=1}^{m} H_{i}\left(b_{i, t}+b_{i, t-L P_{i}+1}\right) \leq L H_{t}, \quad t=1,2, \ldots, n \\
& x_{i, t}=1 \text { if } \mathrm{TR}_{i} \text { is in maintenance in } \mathrm{PR}_{t},=0 \text { otherwise, } \\
& b_{i, t}=1 \text { if the maintenance of } \mathrm{TR}_{i} \text { begins at } \mathrm{PR} t,=0 \text { otherwise, } \\
&
\end{aligned}
$$

in which $x_{i, t}$ and $b_{i, t}$ are both decision variables. The model's target is to obtain a set of turbine maintenance schedules on condition that the system reliability and the maintenance cost are optimised simultaneously with all constraints obeyed. Notably, it is known from Model 27 a, b) that not only objective functions but also constraints are well tailored for offshore wind farms. Two components of the cost criterion (the environmental monitoring cost $C_{i, t}^{E M}$ and the transportation cost $C_{i, t}^{T}$ in Eq. (9)), and six types of constraints (weather, manpower, vehicle, greenhouse gas emission, marine ecosystem, and bird population constraints, see Eqs. (21)-26) are specially formulated for the PM of offshore wind farms.

Remark 1. Eliminating or adjusting some of the costs and constraints that have been implemented particularly for offshore wind farms, a generalized model of Model $(27 \mathrm{a}, \mathrm{b})$ is applicable 
to general power systems.

On the other hand, the uniqueness of Model $(27 \mathrm{a}, \mathrm{b})$ for offshore wind farms can be reflected from differences with the generalised model in wider scope. The convenience of transforming manifests the good applicability and flexibility of Model $(27 \mathrm{a}, \mathrm{b})$, so that we can declare that the PM scheduling model proposed in this paper is reasonable. Furthermore, it is interesting that there exists another common method to represent the reliability maximization objective differently [8]. They define the corresponding objective function as the single objective in their generator maintenance scheduling problem of power systems like

$$
\min _{\boldsymbol{X}} \sum_{t=1}^{n}\left[\sum_{i=1}^{m} p_{i, t}\left(1-x_{i, t}\right)-d_{t}\right]^{2},
$$

which is to quantify the reliability as the sum of squares of the net power reserve (SSR). Thus, the minimisation of the SSR implies the reliability maximization. This definition of the reliability objective is generated from another perspective that the high system reliability implies the little difference among the net power reserves for each time period, namely to make full use of the electric energy and avoid power waste. It is to pursue a high resource utilisation rate.

Therefore, we are going to employ this form of reliability maximisation objective function into our multi-objective, non-linear programming model for PM scheduling of offshore wind farms as well, in order to build a contrast (benchmark) model of Eqs. 27a, b) to compare with the one given by Eq. (28) after converting it into the range [0,1]. To achieve this, we use the weight coefficient $1 / \sum_{t=1}^{n}\left(\sum_{i=1}^{m} p_{i, t}-d_{t}\right)^{2}$ of the SSR $\left[\sum_{i=1}^{m} p_{i, t}\left(1-x_{i, t}\right)-d_{t}\right]^{2}$. Thus, the equivalent form of this different maximisation objective function in Eq. (28) can be indicated as

$$
\min _{\boldsymbol{X}} \sum_{t=1}^{n} \frac{\left[\sum_{i=1}^{m} p_{i, t}\left(1-x_{i, t}\right)-d_{t}\right]^{2}}{\sum_{\tau=1}^{n}\left(\sum_{i=1}^{m} p_{i, \tau}-d_{\tau}\right)^{2}} .
$$

Thus, the contrast model of our problem is constructed by substituting the aforesaid reliability objective Eq. (7) in Eq. (27a) for the minimisation of the SSR Eq. (29), and remaining all the rest objective and constraints unchanged $\sqrt[6]{6}$ In the later section, comparisons and analyses between the maintenance scheduling optimisation model Eqs. (27a, b) and its contrast model for offshore wind farms will be made to have a careful investigation of their performances and characteristics.

\footnotetext{
${ }^{6}$ It should be pointed out that the two values of reliability from Eq. 27a and from Eq. (29) are originated from two different reliability indices, which provide two different interpretations of the reliability ensured by power reserves.
} 


\section{Nondominated Sorting Genetic Algorithm II}

The nondominated sorting genetic algorithm II (NSGA-II) utilised for solving the proposed Model $(27 \mathrm{a}, \mathrm{b})$ for the PM scheduling of offshore wind farms is going to be introduced. Abundant Pareto-optimal solutions can be obtained from the NSGA-II. As none of Pareto-optimal solutions is absolutely better than any other one, each of them is acceptable [21]. Therefore, they can provide various trade-off solutions for determining a satisficing solution to support the decision-making of the offshore wind farm project.

The fast nondominated sorting procedure, the fast crowding distance estimation procedure, and the simple crowded-comparison operator are regarded as three innovations of the NSGA-II, so that weaknesses of NSGA are alleviated to a large extent owing to improvements in aspects of the computational complexity, elitism and diversity preservation. Thus, the whole procedure of the NSGA-II for solving the proposed Model $(27 \mathrm{a}, \mathrm{b})$ is presented in Algorithm 1 in detail (see Appendix). It should be noted that the contrast model is also similarly solved by Algorithm 1 .

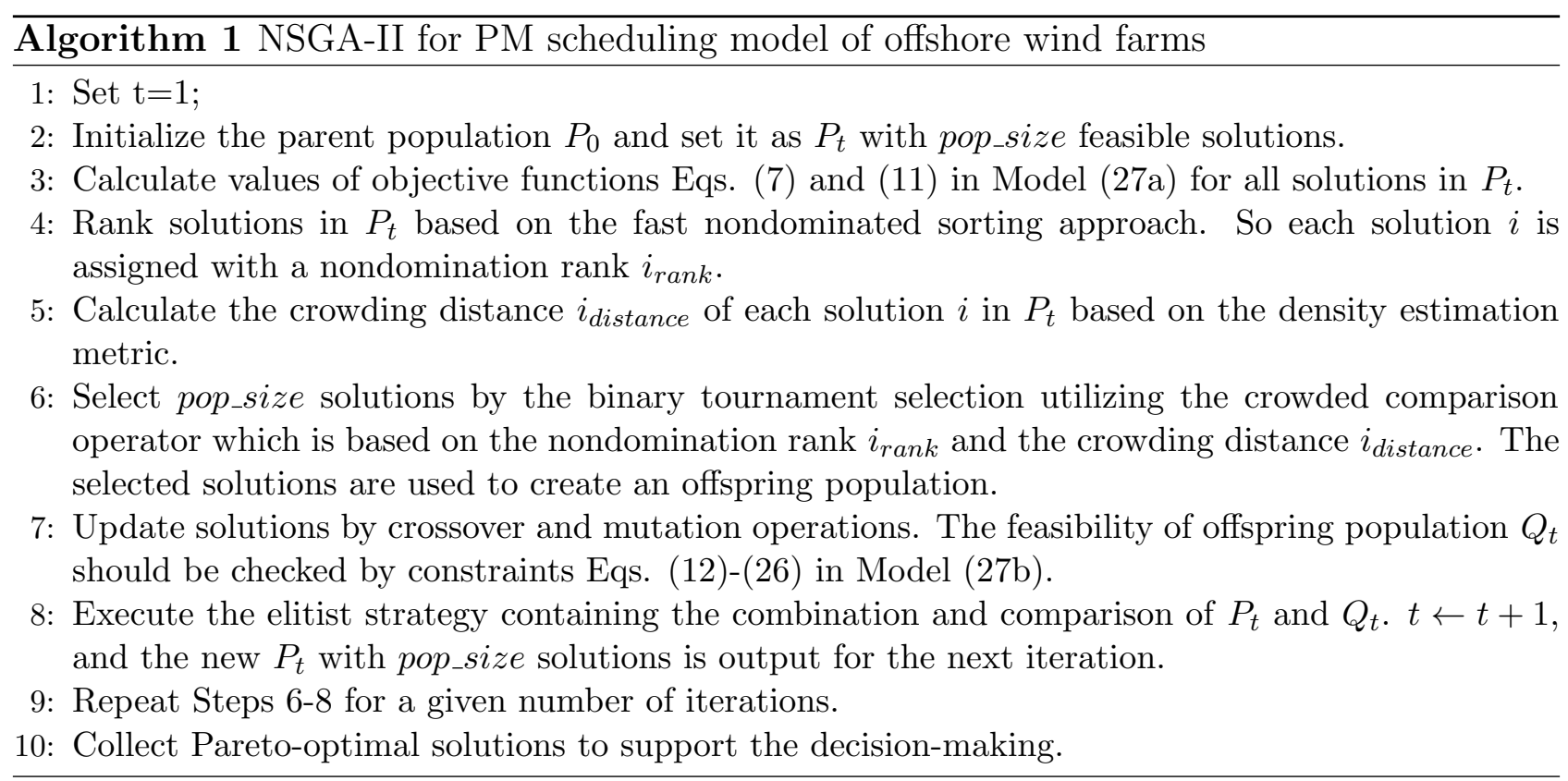

\section{Numerical Example}

In order to verify the feasibility, effectiveness and performance of the proposed constrained non-linear multi-objective programming model for PM scheduling of offshore wind farms, Eqs. (27 $\mathrm{a}, \mathrm{b})$, and its contrast model, as well as the corresponding NSGA-II, a hypothetical case of offshore wind farm preventive maintenance is illustrated as a numerical example. The results are analysed and compared from three main respects in this section. 


\subsection{Background and parameters}

The case we are going to apply and implement is about an offshore wind farm with 50 wind turbines. The time horizon is 52 weeks of a year. Data of the generated energy $p_{i, t}$, the customer power demand $d_{t}$, all maintenance cost components $C_{i, t}^{M}, C_{i, t}^{E Q}, C_{i, t}^{I}, C_{i, t}^{E M}, C_{i, t}^{T}, C_{i, t}^{A}$ and $C_{i, t}^{C R M}$, the maintenance capacity $L T_{t}$, manpower demands $M_{i}^{V}, M_{i}^{H}$ and $M_{i}^{L}$, the available manpower $A M_{t}$, vehicle demands $V_{i}$ and $H_{i}$, available vehicle amounts $A V_{t}$ and $A H_{t}$, all greenhouse gas emission related parameters $z_{i}, q^{V}, q^{H}, \bar{w}, E Q_{i}^{V}, E Q_{i}^{H}$ and $G H G$, navigating vehicle limits $L V_{t}$ and $L H_{t}$ have already been estimated and set reasonably according to historical data and expertise (see Tables B.6 B.10 in Appendix). Besides, the maintenance duration $L P_{i}$ of each turbine is 3 weeks. The maintenance of $\mathrm{TR}_{5}$ is prior to that of $\mathrm{TR}_{16}$. The deadline of $\mathrm{TR}_{27}$ is $\mathrm{PR}_{48}$. The time set not allowed for maintenance is $U=\{1,2,3\}$. Parameter settings for NSGA-II are given in Table B.11 in Appendix.

\subsection{Effects of different decision-maker's attitudes}

As there are mainly four different kinds of decision-maker's attitudes towards the wind farm project over the time horizon, i.e., fully rational, optimism biased, wait-and-see and pessimism biased preferences, their different impacts on final solutions are shown in this section. It essentially means we need to assign attainment exponents $s_{1}, s_{2}, \ldots, s_{52}$ by various combinations of $s_{t}$ with positive, neutral or negative effects in our case.

Table 3: Assignment of attainment exponents $s_{t}$ into different decision-maker's attitudes

\begin{tabular}{cccccc|cccccc}
\hline$s_{t}$ & Rat & Opt & W\&s & Pes $^{1}$ & Pes $^{2}$ & $s_{t}$ & Rat & Opt & W\&s & Pes $^{1}$ & Pes $^{2}$ \\
\hline$s_{1}$ & 0.21 & 0.58 & 1 & 47.50 & 2 & $s_{27}$ & 1 & 0.03 & 1 & 2.06 & 2 \\
$s_{2}$ & 0.29 & 0.12 & 1 & 19.21 & 2 & $s_{28}$ & 1 & 0.24 & 1 & 17.95 & 2 \\
$s_{3}$ & 0.70 & 0.41 & 1 & 9.78 & 2 & $s_{29}$ & 1 & 0.97 & 1 & 23.91 & 2 \\
$s_{4}$ & 0.71 & 0.67 & 1 & 15.47 & 2 & $s_{30}$ & 1 & 0.15 & 1 & 2.22 & 2 \\
$s_{5}$ & 0.72 & 0.68 & 1 & 4.85 & 2 & $s_{31}$ & 1 & 0.84 & 1 & 7.84 & 2 \\
$s_{6}$ & 0.23 & 0.01 & 1 & 3.44 & 2 & $s_{32}$ & 1 & 0.48 & 1 & 39.87 & 2 \\
$s_{7}$ & 0.84 & 0.81 & 1 & 10.17 & 2 & $s_{33}$ & 1 & 0.07 & 1 & 8.92 & 2 \\
$s_{8}$ & 0.93 & 0.70 & 1 & 32.29 & 2 & $s_{34}$ & 1 & 0.40 & 1 & 5.05 & 2 \\
$s_{9}$ & 0.41 & 0.17 & 1 & 35.85 & 2 & $s_{35}$ & 14.04 & 0.66 & 1 & 40.52 & 2 \\
$s_{10}$ & 0.69 & 0.61 & 1 & 27.68 & 2 & $s_{36}$ & 40.11 & 0.78 & 1 & 27.84 & 2 \\
$s_{11}$ & 0.25 & 0.29 & 1 & 38.26 & 2 & $s_{37}$ & 23.70 & 0.33 & 1 & 22.65 & 2 \\
$s_{12}$ & 0.79 & 0.37 & 1 & 4.26 & 2 & $s_{38}$ & 19.45 & 0.74 & 1 & 28.53 & 2 \\
$s_{13}$ & 0.96 & 0.72 & 1 & 6.63 & 2 & $s_{39}$ & 49.97 & 0.44 & 1 & 43.74 & 2 \\
$s_{14}$ & 0.53 & 0.27 & 1 & 11.81 & 2 & $s_{40}$ & 39.96 & 0.02 & 1 & 22.35 & 2 \\
$s_{15}$ & 0.95 & 0.11 & 1 & 24.01 & 2 & $s_{41}$ & 3.63 & 0.23 & 1 & 40.59 & 2 \\
$s_{16}$ & 0.01 & 0.06 & 1 & 18.02 & 2 & $s_{42}$ & 45.28 & 0.86 & 1 & 5.31 & 2 \\
$s_{17}$ & 0.12 & 0.06 & 1 & 18.60 & 2 & $s_{43}$ & 46.61 & 0.79 & 1 & 7.99 & 2 \\
$s_{18}$ & 0.40 & 0.04 & 1 & 47.53 & 2 & $s_{44}$ & 23.06 & 0.07 & 1 & 4.51 & 2 \\
$s_{19}$ & 1 & 0.06 & 1 & 16.15 & 2 & $s_{45}$ & 36.72 & 0.48 & 1 & 2.40 & 2 \\
$s_{20}$ & 1 & 0.16 & 1 & 20.32 & 2 & $s_{46}$ & 28.96 & 0.91 & 1 & 40.40 & 2 \\
$s_{21}$ & 1 & 0.78 & 1 & 14.56 & 2 & $s_{47}$ & 36.90 & 0.82 & 1 & 9.90 & 2 \\
$s_{22}$ & 1 & 0.19 & 1 & 40.26 & 2 & $s_{48}$ & 15.47 & 0.60 & 1 & 35.70 & 2 \\
$s_{23}$ & 1 & 0.17 & 1 & 1.45 & 2 & $s_{49}$ & 9.09 & 0.48 & 1 & 4.38 & 2 \\
$s_{24}$ & 1 & 0.44 & 1 & 29.57 & 2 & $s_{50}$ & 6.96 & 0.07 & 1 & 35.08 & 2 \\
$s_{25}$ & 1 & 0.97 & 1 & 10.37 & 2 & $s_{51}$ & 42.39 & 0.87 & 1 & 30.01 & 2 \\
$s_{26}$ & 1 & 0.69 & 1 & 47.58 & 2 & $s_{52}$ & 21.46 & 0.56 & 1 & 46.84 & 2 \\
\hline
\end{tabular}

First, we allocate all 52 attainment exponents to four types of attitudes as shown in Table 3. As to the fully rational attitude (2nd and 8th columns), we select $s_{1}, s_{2}, \ldots, s_{18}$ randomly from 


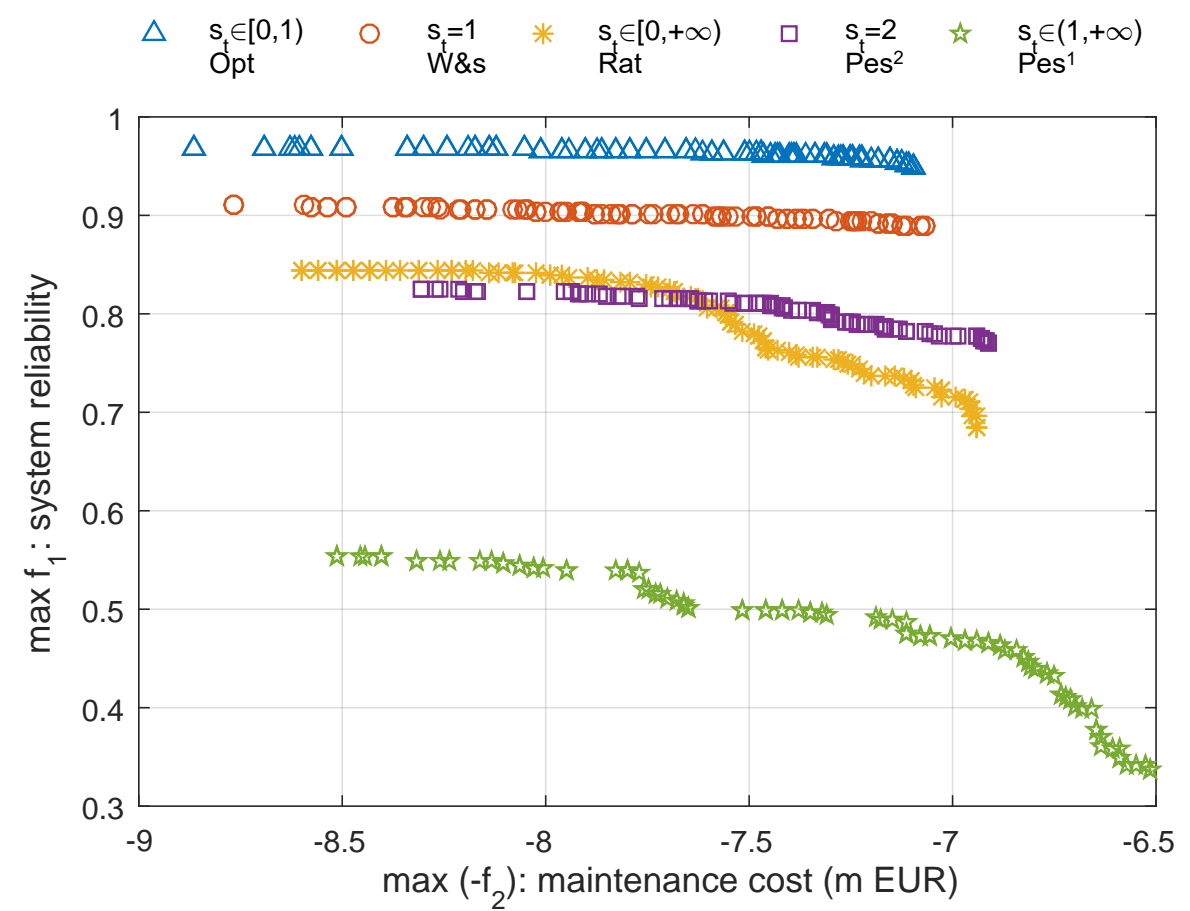

Fig. 2: Effects of different decision-maker's attitudes on solutions.

Table 4: Ranges of two objectives on different decision-maker's attitudes

\begin{tabular}{ccccc}
\hline Attitude & Reliability $^{L}$ & Reliability $^{U}$ & Cost $^{L}(\mathrm{~m} €)$ & Cost $^{U}(\mathrm{~m} €)$ \\
\hline Rational & 0.684 & 0.845 & 6.939 & 8.600 \\
Optimistic & 0.949 & 0.968 & 7.096 & 8.862 \\
W\&s & 0.888 & 0.910 & 7.069 & 8.765 \\
Pessimistic $^{1}$ & 0.338 & 0.553 & 6.512 & 8.511 \\
Pessimistic $^{2}$ & 0.770 & 0.824 & 6.914 & 8.305 \\
\hline
\end{tabular}


$[0,1)$, make $s_{19}, s_{20}, \ldots, s_{34}$ all equal to 1 , and choose $s_{35}, s_{36}, \ldots, s_{52}$ randomly from $(1,50)$,

which can be approximately equivalent to the interval $(1,+\infty)$. For the optimism biased attitude (3rd and 9th columns), $s_{1}, s_{2}, \ldots, s_{52}$ are entirely from [0,1). For the wait-and-see attitude (4th and 10th columns), all $s_{t}$ are equal to 1, which means no exponents exist and the same situation with that of the first conventional reliability criterion (the power reserve ratio). For the pessimism biased attitude, $s_{1}, s_{2}, \ldots, s_{52}$ in the 5th and 11th columns are randomly picked from $(1,50)$, while those in the 6 th and 12 th columns are all set as $s_{t}=2$, which is a special case for further comparison with the contrast model containing quadratic terms.

All five multi-objective programming models (based on Eqs. (27a, b)) are implemented for 5000 iterations, respectively and the final solutions are displayed in Fig. 2 by five different point types and also in Table 4. It is apparent from Fig. 2 and more precisely from Table 4 that results on the basis of the fully rational attitude (yellow asterisks in Fig. 2 and 2nd row in Table 4) have the best spread of Pareto-optimal solutions, which can provide much wider and more distinguishable choices both on system reliability (changes from 0.684 to 0.845 ) and maintenance cost (from $€ 6.939 \mathrm{~m}$ to $€ 8.600 \mathrm{~m}$ ) directions for trading-off and supporting the decision-making. Although solutions with optimistic, wait-and-see and pessimistic $\left(s_{t}=2\right)$ attitudes achieve extremely high values of reliability, their solution sets contain a few gaps and their spreads are relatively narrow and partial on the reliability axis. Moreover, solutions with the pessimistic attitude $\left(s_{t}>1\right)$ form good spreads on both of the reliability and the cost axes, but their values of reliability are relatively too low (even the upper bound Reliability $=0.553$ ). Hence, we can conclude that the fully rational attitude seems more appropriate for decisionmakers to hold because it not only offers more diverse options, but also makes the results more reasonable and effective.

Thus, in the following analyses, we will primarily focus on the multi-objective optimization model with attainment exponents setting based on the fully rational attitude. Obviously, this is not an exhaustive and conclusive way, as different decision-makers can take an alternative strategy as the most preferable one. Actually, the model flexibility is one of the main advantage of our treatment.

\subsection{Solutions and guidance for decision-making}

In this section, we will analyse in detail the Pareto-optimal solutions of the proposed model (Eqs. (27a, b)), in order to provide a practical guidance for decision-making on the PM scheduling problem of offshore wind farms. Values of attainment exponents $s_{t}$ are assigned according to the fully rational attitude, i.e., and the same with values in the 2 nd and 8th columns of 
Table 3. In Fig. 3, asterisks represent Pareto-optimal solutions after 5000 iterations. We can

extract some decision instructions aiming at different strategic environments of an offshore wind farm project as follows:

(1) If the offshore wind farm project executes a cost priority strategy, it means that decision-

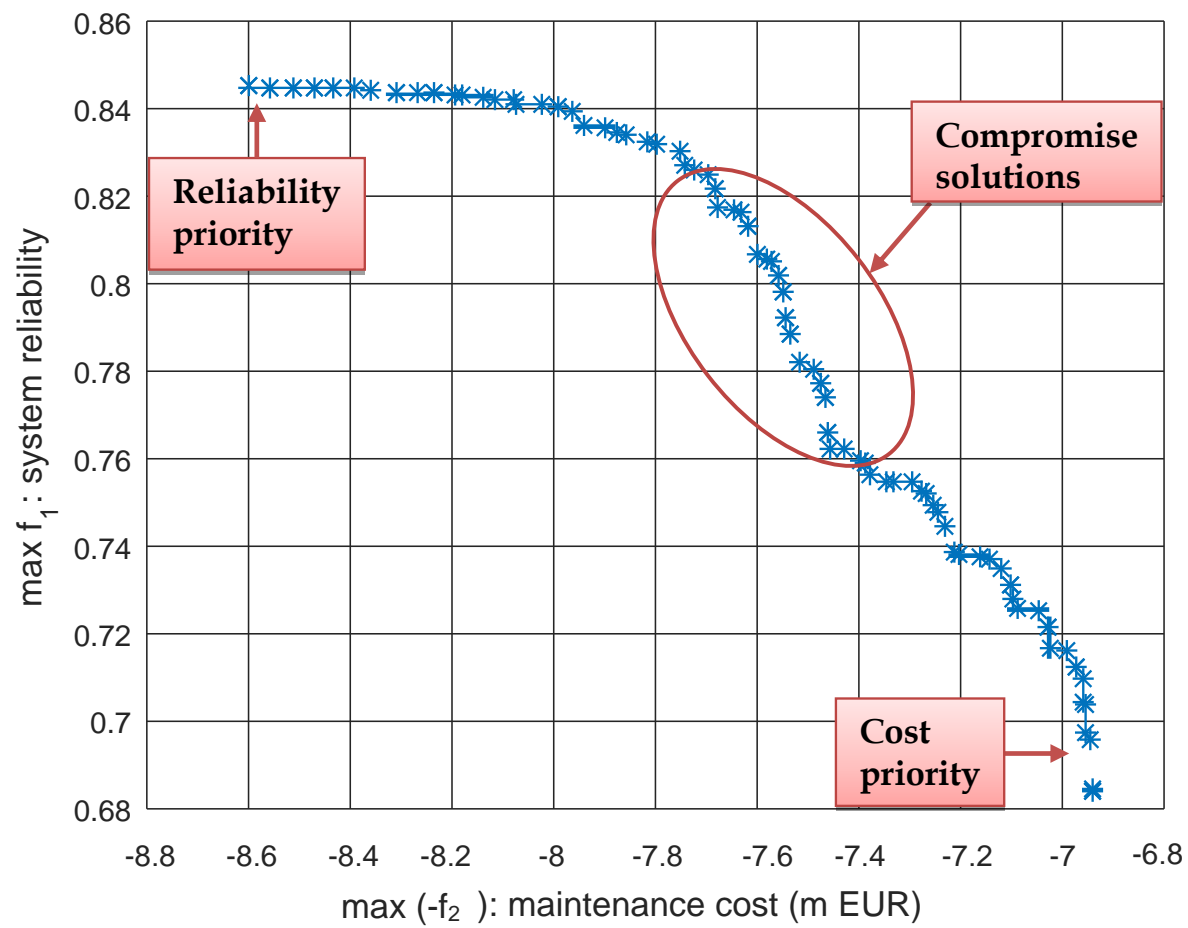

Fig. 3: Pareto-optimal solutions after 5000 iterations with the fully rational attitude.

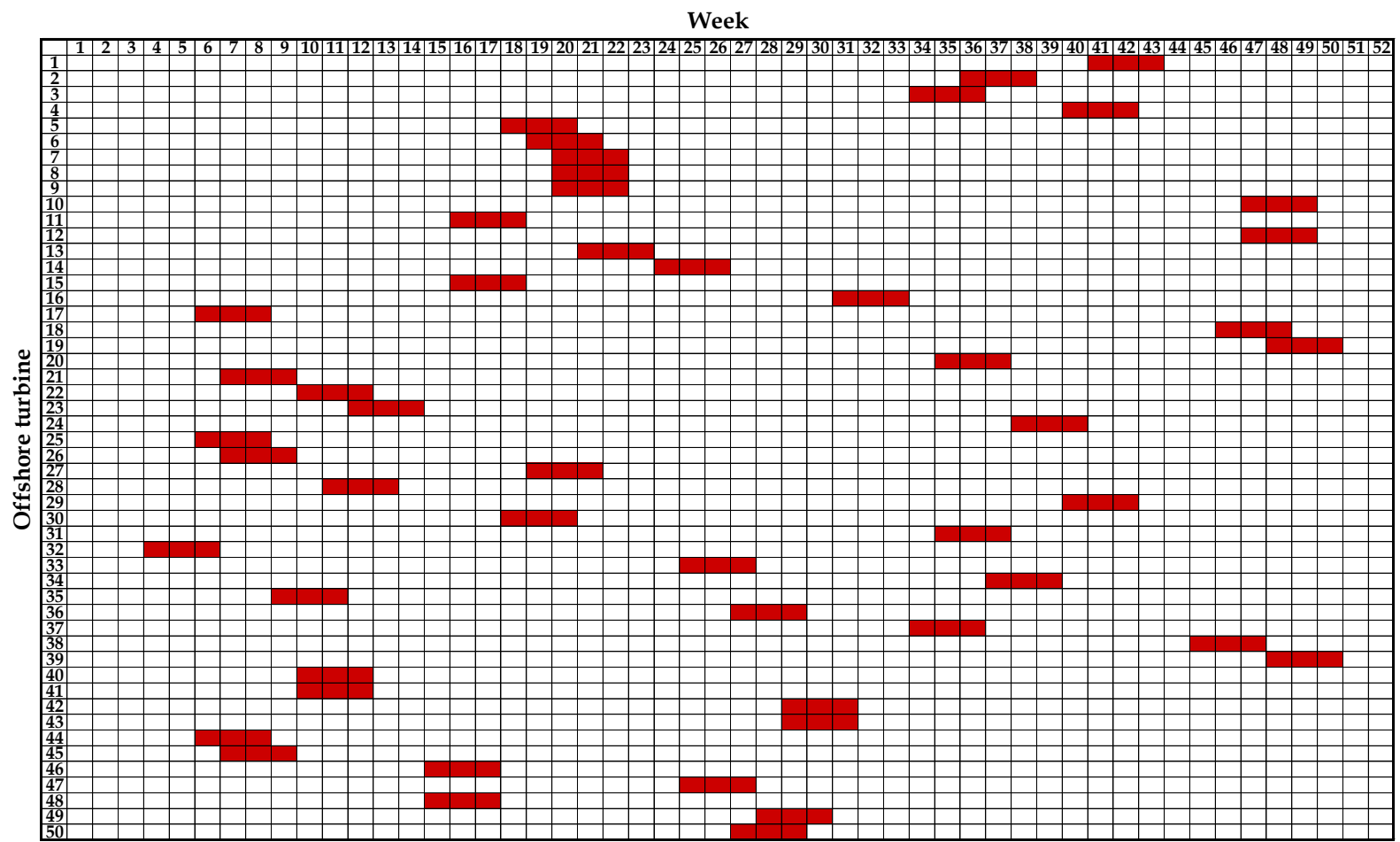

(a) PM schedule of a cost priority solution.

Fig. 4: Example Schedules of different Pareto-optimal solutions. 


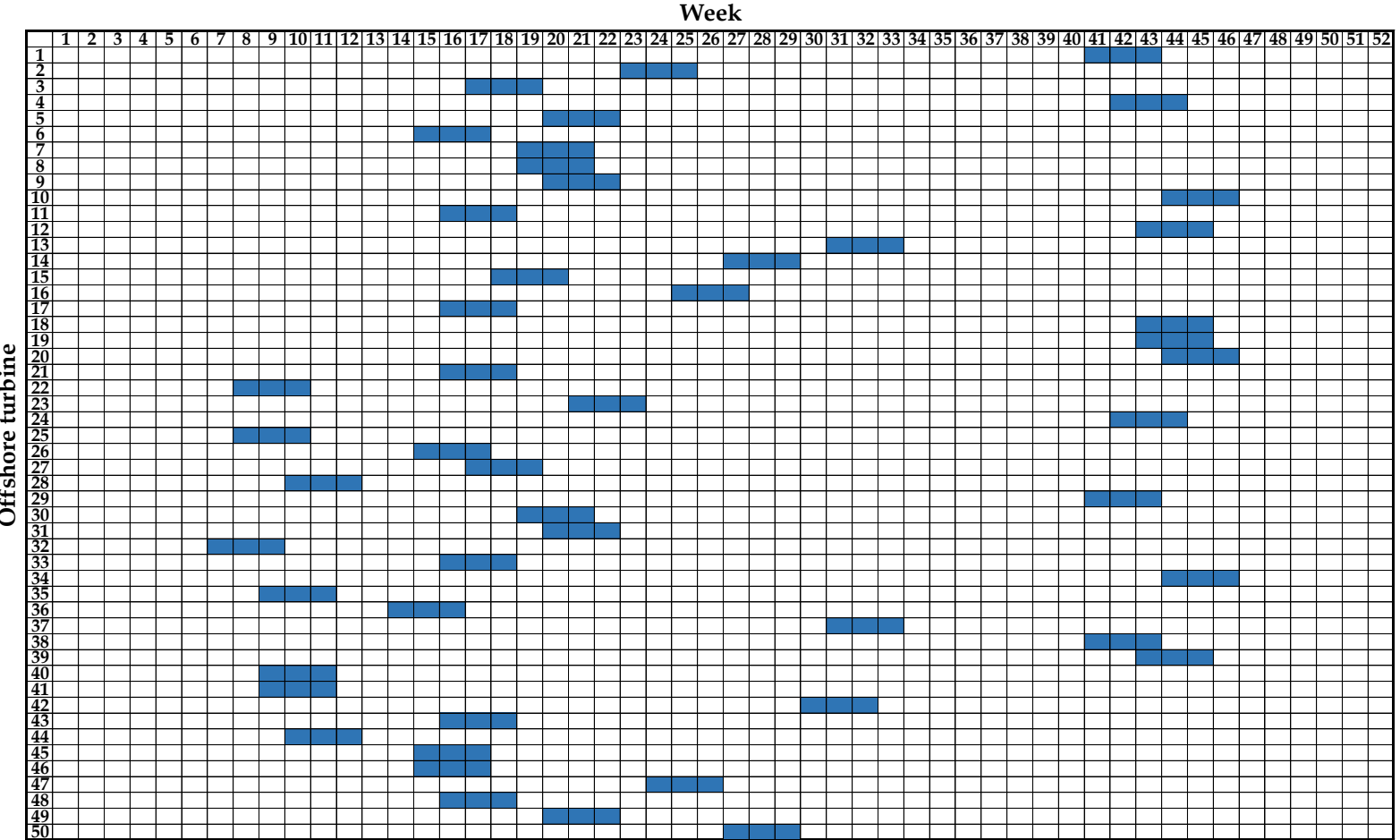

(b) PM schedule of a reliability priority solution.

Week

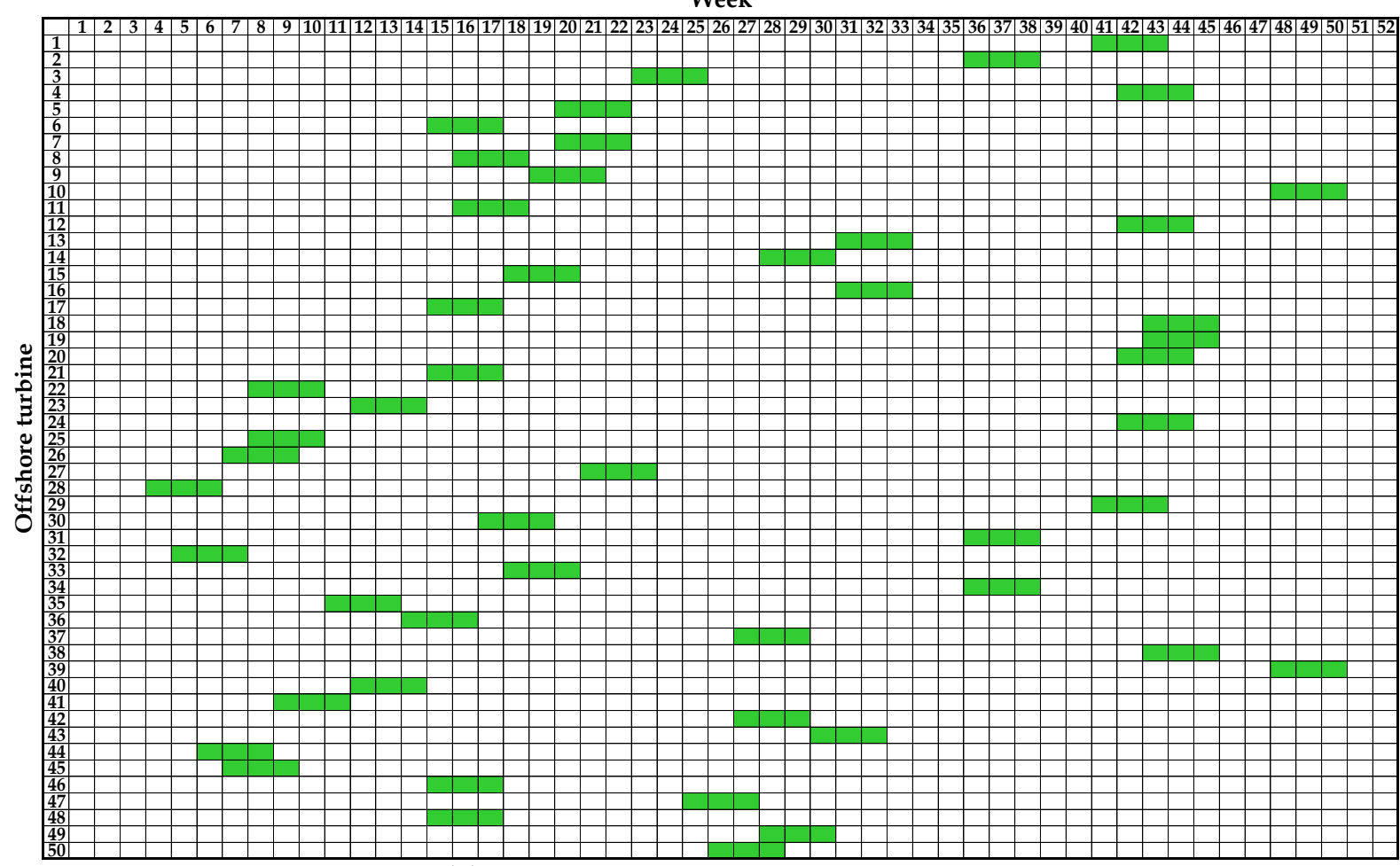

(c) PM schedule of a compromise solution.

Fig. 4: Example Schedules of different Pareto-optimal solutions. 
Table 5: Examples of Pareto-optimal solutions for different strategic environments

(a) Pareto-optimal solutions for cost priority strategy

\begin{tabular}{ccc|ccc}
\hline Solution & Reliability & Cost $(\mathrm{m} €)$ & Solution & Reliability & Cost $(\mathrm{m} €)$ \\
\hline 1 & 0.684 & 6.939 & 4 & 0.713 & 6.972 \\
2 & 0.696 & 6.943 & 5 & 0.722 & 7.027 \\
3 & 0.704 & 6.954 & 6 & 0.731 & 7.101 \\
\hline
\end{tabular}

(b) Pareto-optimal solutions for reliability priority strategy

\begin{tabular}{ccc|ccc}
\hline Solution & Reliability & Cost $(\mathrm{m} €)$ & Solution & Reliability & Cost $(\mathrm{m} €)$ \\
\hline 1 & 0.845 & 8.600 & 4 & 0.841 & 8.025 \\
2 & 0.844 & 8.359 & 5 & 0.840 & 7.991 \\
3 & 0.843 & 8.194 & 6 & 0.836 & 7.942 \\
\hline
\end{tabular}

(c) Pareto-optimal solutions for compromise strategy

\begin{tabular}{ccc|ccc}
\hline Solution & Reliability & Cost $(\mathrm{m} €)$ & Solution & Reliability & Cost $(\mathrm{m} €)$ \\
\hline 1 & 0.762 & 7.428 & 4 & 0.798 & 7.549 \\
2 & 0.774 & 7.466 & 5 & 0.806 & 7.601 \\
3 & 0.788 & 7.536 & 6 & 0.818 & 7.676 \\
\hline
\end{tabular}

makers put the maintenance cost as the first consideration and want to save as much as possible. To pursue low cost implies to sacrifice the achievement of the system reliability. As long as the reliability is not so low that it will influence the basic stability, decision-makers are willing to adopt a solution with the cost close to the lowest and the low but acceptable reliability. For example, the solution with the lowest cost as $€ 6.939 \mathrm{~m}$ and reliability as 0.684 among all results, i.e., the asterisk on the bottom right corner in Fig. 3, can be chosen as a decision of cost priority. The corresponding maintenance schedule of this solution is shown in Fig. 4a, in which the blocks refer to periods in maintenance. In addition, five other cost priority solutions are given in Table 5 a.

(2) If the offshore wind farm project carries out a reliability priority strategy, which implies that the customer demand satisfaction is more significant to decision-makers and they have sufficient investments so that the maintenance budget is not a significant problem, Pareto-optimal solutions in the top left corner of Fig. 3 are their best choices. As long as the maintenance cost does not exceed the budget, higher reliability level can be aspired. The upper bound decision with the highest reliability can be easily found in the figure. It reaches the reliability as 0.845 and the cost as $€ 8.600 \mathrm{~m}$ as a compensation. The corresponding maintenance schedule of this solution is shown in Fig. 4b, Also, five other reliability priority solutions are listed in Table 5b. It is notable that the blocks are concentrated in relatively early periods and there are no more turbines in maintenance from $\mathrm{PR}_{34}$ to $\mathrm{PR}_{40}$ and since $\mathrm{PR}_{47}$. The reason for this phenomenon is that when decision-makers hold the fully rational attitude, settings of attainment exponents $s_{t}$ with this attitude have already decided that the high reliability signifies maintaining as early as possible. Differences in distributions of schedules tend to be the most obvious between two solutions with the lower bound of cost and the upper bound of reliability which can be observed 
from Fig. $4 \mathrm{a}$ and Fig. $4 \mathrm{~b}$.

(3) If both the maintenance cost and the system reliability are important and almost unbiased to the project strategy in eyes of decision-makers, some compromise solutions should be considered. Compromise solutions mean those not sacrificing a lot on the optimization of either objective function, so which also implies a particularly outstanding optimised direction can also not be reached among these solutions. They are marked in the circle in Fig. 3, and six compromise solutions are listed in Table $5 \mathrm{c}$. The maintenance schedule of the first solution in Table $5 \mathrm{c}$ is indicated in Fig. 4c. It can be seen that the distribution of the schedule in Fig. $4 \mathrm{c}$ has less obvious centralised tendency than those in Fig. 4a and Fig. 4b.

To sum up, it can be seen that no matter what strategy the offshore wind farm project adopts, Pareto-optimal solutions obtained from the optimisation are able to provide adequate alternative satisfying solutions to decision-makers. According to Porter's generic strategies [38], when decision-makers take adopt a cost leadership strategy for offshore wind farm maintenance especially in the early time of the project, a decision can be selected from solutions in the bottom right corner of Fig. 3. When the execution of the project begins to stabilise, the differentiation strategy (i.e., the customer-oriented strategy) is more likely to be adopted in order to satisfy customer needs for more profits. On this occasion, the primary mission is to pursue high reliability, which means to make a decision from the solutions in the top left corner of Fig. 3. When the focus strategy is taken to consider the cost and the customer satisfaction simultaneously, decision-makers are not partial to either of the two objectives. A decision to support this coordination strategy can be made from solutions in the circle of Fig. 3. The maintenance schedule solutions corresponding to a certain maintenance strategy (cost leadership, differentiation, or focus strategies) can be timely and newly obtained by implementing the model and algorithm again after constraining those wind turbines that already completed the maintenance jobs in the past periods, whenever the decision-maker determines to switch to a different strategy from the present one at any period during the time horizon.

\subsection{Comparisons between the two reliability objectives}

In this section, we make comparisons between two approaches (i.e., the reliability maximisation and the SSR minimisation) of the system reliability maximisation objective in the proposed model, Eqs. (27a, b), and its contrast model. Comparisons are made twice, one is the proposed model with the fully rational attitude vs. the contrast model, and the other is the proposed model with attainment exponents $s_{t}=2$ vs. the contrast model because it contains quadratic terms. 
Results of the two comparisons are shown in the following Fig. 5a and Fig. 5b, which are found to be almost similar. It should also be noted that in both figures, the left vertical axis is for Model $(27 \mathrm{a}, \mathrm{b})$ and the right vertical axis is for its contrast model. Hence, some synthetical conclusions can be drawn from the two figures:

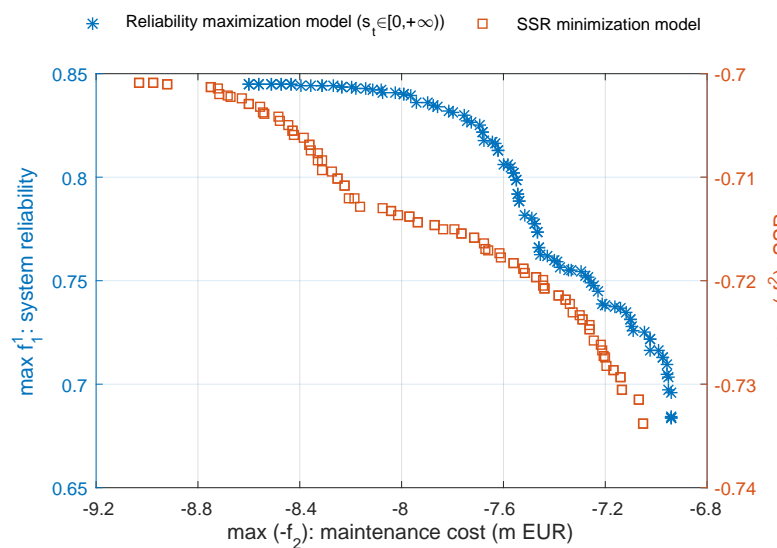

(a) Proposed model with fully rational attitude vs. its contrast model.

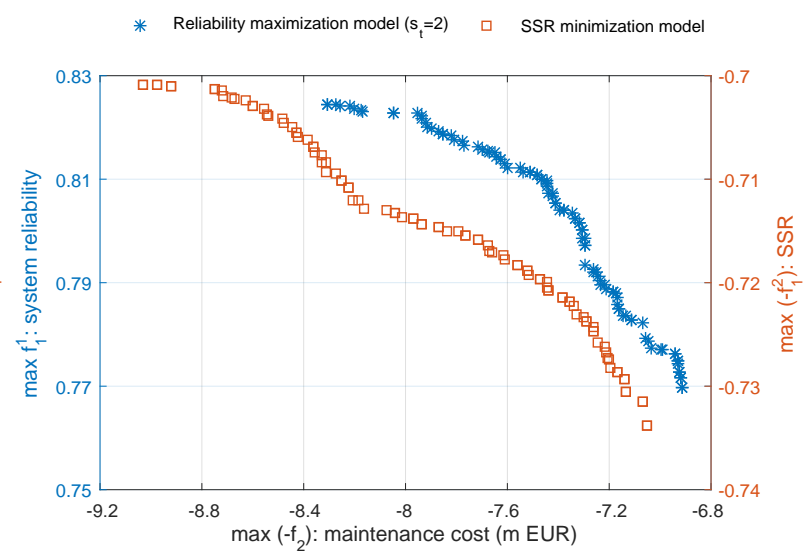

(b) Proposed model with $s_{t}=2$ vs. the contrast model.

Fig. 5: Comparisons between two reliability objectives.

(1) The maintenance cost of Model $(27 \mathrm{a}, \mathrm{b})$ can achieve lower results than that of the contrast model, and some high values of the cost that the contrast model includes are not in the value range of Model $(27 \mathrm{a}, \mathrm{b})$. Consequently, Model $(27 \mathrm{a}, \mathrm{b})$ has an obvious cost advantage over its contrast model.

(2) With respect to the system reliability, it can be seen from Fig. 5a that the range of the reliability distribution of Model $(27 \mathrm{a}, \mathrm{b})$ (approximately 0.16 ) is much wider than that of the contrast model (approximately 0.03), which means Model $(27 \mathrm{a}, \mathrm{b})$ can offer a better decision support and more reliability choices than its contrast model.

\section{Conclusions}

In this paper, we contribute to the corresponding literature in the following four ways: (i) we optimise the reliability and cost objectives simultaneously in the PM scheduling problem with the background of offshore wind farms, making the problem more comprehensive and closer to reality; (ii) we propose a new definition of the reliability criterion by utilising an attainment exponent which can be regarded as an expansion of previous definitions; (iii) we also well design the components of the maintenance cost criterion and constraints particularly applicable to the offshore wind farm environment; (iv) we employ the NSGA-II to solve our constrained non-linear multi-objective programming model for the PM scheduling of offshore wind farms, and obtain a set of Pareto-optimal solutions for supporting decision-making. 


\section{Acknowledgments}

The authors would like to thank the anonymous reviewers for their insightful comments that significantly improved the quality of this paper. Moreover, the gracious supports of the EPSRC and ESRC Centre for Doctoral Training on Quantification and Management of Risk and Uncertainty in Complex Systems and Environment (EP/L015927/1), the Recruitment Program of High-end Foreign Experts (Grant No. GDW20163100009), and the China Scholarship Council ([2014]3026) should be acknowledged.

\section{References}

[1] M. Ben-Daya, S.O. Duffuaa, A. Raouf, Maintenance, modeling and optimization, Norwell, Massachusetts: Klüwer. (2000).

[2] S.P. Canto, Application of Benders' decomposition to power plant preventive maintenance scheduling, European Journal of Operational Research. 184(2) (2008) 759-777.

[3] S.P. Canto, Using 0/1 mixed integer linear programming to solve a reliability-centered problem of power plant preventive maintenance scheduling, Optimization and Engineering. 12(3) (2011) 333-347.

[4] S.P. Canto, J.C.R. Romero, A model for the preventive maintenance scheduling of power plants including wind farms, Reliability Engineering \& System Safety. 119 (2013) 67-75.

[5] D. Chattopadhyay, Life-cycle maintenance management of generating units in a competitive environment, IEEE Transactions on Power Systems. 19(2) (2004) 1181-1189.

[6] A.J. Conejo, R. García-Bertrand, M. Díaz-Salazar, Generation maintenance scheduling in restructured power systems, IEEE Transactions on Power Systems. 20(2) (2005) 984-992.

[7] D. Cyranoski, Beijing's windy bet, Nature. 457 (2009) 372-374.

[8] K.P. Dahal, C.J. Aldridge, J.R. McDonald, Generator maintenance scheduling using a genetic algorithm with a fuzzy evaluation function, Fuzzy Sets and Systems. 102(1) (1999) 21-29.

[9] K.P. Dahal, K. Al-Arfaj, K. Paudyal, Modelling generator maintenance scheduling costs in deregulated power markets, European Journal of Operational Research. 24(2) (2015) $551-561$. 
[10] K.P. Dahal, N. Chakpitak, Generator maintenance scheduling in power systems using

[11] K.P. Dahal, S.J. Galloway, Evolutionary generator maintenance scheduling in power systems, Evolutionary Scheduling, Springer Berlin Heidelberg. (2007) 349-382.

[12] Y. Dalgic, I. Lazakis, I. Dinwoodie, et al., Advanced logistics planning for offshore wind farm operation and maintenance activities, Ocean Engineering. 101 (2015) 211-226.

[13] K. Deb, A. Pratap, S. Agarwal, et al., A fast and elitist multiobjective genetic algorithm: NSGA-II, IEEE Transactions on Evolutionary Computation. 6(2) (2002) 182-197.

[14] F. Ding, Z. Tian, Opportunistic maintenance for wind farms considering multi-level imperfect maintenance thresholds, Renewable Energy. 45 (2012) 175-182.

[15] M. Doostparast, F. Kolahan, M. Doostparast, A reliability-based approach to optimize preventive maintenance scheduling for coherent systems, Reliability Engineering \& System Safety. 126 (2014) 98-106.

[16] G.T. Egan, T.S. Dillon, K. Morsztyn, An experimental method of determination of optimal maintenance schedules in power systems using the branch-and-bound technique, IEEE Transactions on Systems, Man, and Cybernetics. 6(8) (1976) 538-547.

[17] U.E. Ekpenyong, J. Zhang, X. Xia, An improved robust model for generator maintenance scheduling, Electric Power Systems Research. 92 (2012) 29-36.

[18] M.Y. El-Sharkh, Clonal selection algorithm for power generators maintenance scheduling, International Journal of Electrical Power \& Energy Systems. 57 (2014) 73-78.

[19] M. Fattahi, M. Mahootchi, H. Mosadegh, et al., A new approach for maintenance scheduling of generating units in electrical power systems based on their operational hours, Computers \& Operations Research. 50 (2014) 61-79.

[20] C. Feng, X. Wang, J. Wang, Iterative approach to generator maintenance schedule considering unexpected unit failures in restructured power systems, European Transactions on Electrical Power. 21(1) (2011) 142-154.

[21] C.M. Fonseca, P.J. Fleming, An overview of evolutionary algorithms in multiobjective optimization, Evolutionary Computation. 3(1) (1995) 1-16. 
[22] S. Ghanmi, M. Guedri, M.L. Bouazizi, et al., Robust multi-objective and multi-level optimization of complex mechanical structures, Mechanical Systems and Signal Processing. 25 (2011) 2444-2461.

[23] C. Gundegjerde, I.B. Halvorsen, E.E. Halvorsen-Weare, et al., A stochastic fleet size and mix model for maintenance operations at offshore wind farms, Transportation Research Part C: Emerging Technologies. 52 (2015) 74-92.

[24] G.G. Hassan, A guide to UK offshore wind operations and maintenance, Scottish Enterprise and the Crown Estate. (2013).

[25] C.A. Irawan, D. Ouelhadj, D. Jones, et al., Optimisation of maintenance routing and scheduling for offshore wind farms, European Journal of Operational Research. 256(1) (2017) 76-89.

[26] A. Karyotakis, On the optimisation of operation and maintenance strategies for offshore wind farms, Doctoral dissertation, UCL (University College London). (2011).

[27] B.L. Kralj, R. Petrović, Optimal preventive maintenance scheduling of thermal generating units in power systems - a survey of problem formulations and solution methods, European Journal of Operational Research. 35(1) (1988) 1-15.

[28] N.D. Lagaros, M.G. Karlaftis , M.K. Paida, Stochastic life-cycle cost analysis of wind parks, Reliability Engineering \& System Safety. 144 (2015) 117-127.

[29] R.C. Leou, A flexible unit maintenance scheduling considering uncertainties, IEEE Transactions on Power Systems. 16(3) (2001) 552-559.

[30] R.C. Leou, A new method for unit maintenance scheduling considering reliability and operation expense, International Journal of Electrical Power \& Energy Systems. 28(7) (2006) 471-481.

[31] X. Li, D. Ouelhadj, X. Song, et al., A decision support system for strategic maintenance planning in offshore wind farms, Renewable Energy. 99 (2016) 784-799.

[32] S. Luce, Choice criteria in conditional preventive maintenance, Mechanical Systems and Signal Processing. 13(1) (1999) 163-168.

[33] A. Lucifredi, C. Mazzieri, M. Rossi, Application of multiregressive linear models, dynamic Kriging models and neural network models to predictive maintenance of hydroelectric power systems, Mechanical Systems and Signal Processing. 14(3) (2000) 471-494. 
[34] C. Lv, J. Wang, S. You, et al., Short-term transmission maintenance scheduling based on the Benders decomposition, International Transactions on Electrical Energy Systems. $25(4)(2015) 697-712$.

[35] M.K.C. Marwali, S.M. Shahidehpour, Integrated generation and transmission maintenance scheduling with network constraints, IEEE Transactions on Power Systems. 13(3) (1998) 1063-1068.

[36] T. Michler-Cieluch, G. Krause, B.H. Buck, Reflections on integrating operation and maintenance activities of offshore wind farms and mariculture, Ocean \& Coastal Management. $52(1)(2009)$ 57-68.

[37] M. Mollahassani-Pour, A. Abdollahi, M. Rashidinejad, Application of a novel cost reduction index to preventive maintenance scheduling, International Journal of Electrical Power \& Energy Systems. 56 (2014) 235-240.

[38] M.E. Porter, Competitive strategy: Techniques for analyzing industries and competition, New York. (1980).

[39] G. Russo, Renewable energy: Wind power tests the waters, Nature. 513 (2014) 478-480.

[40] B.R. Sarker, T.I. Faiz, Minimizing maintenance cost for offshore wind turbines following multi-level opportunistic preventive strategy, Renewable Energy. 85 (2016) 104-113.

[41] J.D. Schaffer, Multiple objective optimization with vector evaluated genetic algorithms, In: Genetic algorithms and their applications, Proceedings of the 1st International Conference on Genetic Algorithms, Pittsburgh, PA, USA. (1985) 93-100.

[42] Q. Schiermeier, Germany's energy gamble, Nature. 496 (2013) 156-158.

[43] E.B. Schlünz, J.H. Van Vuuren, An investigation into the effectiveness of simulated annealing as a solution approach for the generator maintenance scheduling problem, International Journal of Electrical Power \& Energy Systems. 53 (2013) 166-174.

[44] C. P. Simon, L. Blume, Mathematics for Economists. W. W. Norton \& Company, New York (1994).

[45] N. Srinivas, K. Deb, Muiltiobjective optimization using nondominated sorting in genetic algorithms, Evolutionary Computation. 2(3) (1994) 221-248. 
[46] The Crown Estate, Offshore Wind: Operational Report. (2015) 1-20.

[47] The European Wind Energy Association (EWEA), The European Offshore Wind Industry - Key Trends and Statistics. (2015) 1-12.

[48] Y. Wang, H. Pham, A multi-objective optimization of imperfect preventive maintenance policy for dependent competing risk systems with hidden failure, IEEE Transactions on Reliability. 60(4) (2011) 770-781.

[49] F. Yang, C.S. Chang, Multiobjective evolutionary optimization of maintenance schedules and extents for composite power systems, IEEE Transactions on Power Systems. 24(4) (2009) 1694-1702.

[50] F. Yang, C.M. Kwan, C.S. Chang, Multiobjective evolutionary optimization of substation maintenance using decision-varying Markov model, IEEE Transactions on Power Systems. 23(3) (2008) 1328-1335.

[51] J.P. Zhan, C.X. Guo, Q.H. Wu, et al., Generation maintenance scheduling based on multiple objectives and their relationship analysis, Journal of Zhejiang University SCIENCE C. 15(11) (2014) 1035-1047.

[52] Z. Zhang, A. Kusiak, Z. Song, Scheduling electric power production at a wind farm, European Journal of Operational Research. 224(1) (2013) 227-238. 


\section{Appendix A. Nondominated Sorting Genetic Algorithm II [13]}

\section{Appendix A.1. Fast nondominated sorting procedure}

For each individual $p$ in the population of size $N$, two entities are calculated: (1) $S_{p}$, a set of individuals that the individual $p$ dominates, and (2) domination count $n_{p}$, the number of individuals which dominate the individual $p$.

All individuals in the first nondominated front will have their domination count as zero. Now, for each individual $p$ in the first front, we visit each member $q$ of its set $S_{p}$ and reduce its domination count by one. In doing so, if for any member $q$, the domination count becomes zero, we put it in a separate list $Q$. These members belong to the second nondominated front. Next, the above procedure is continued with each member of $Q$ and the third front is identified. This process continues until all fronts are identified. Thus, the pseudocode of fast nondominated sorting approach which requires $O\left(m N^{2}\right)$ computations are shown in Algorithm 2.

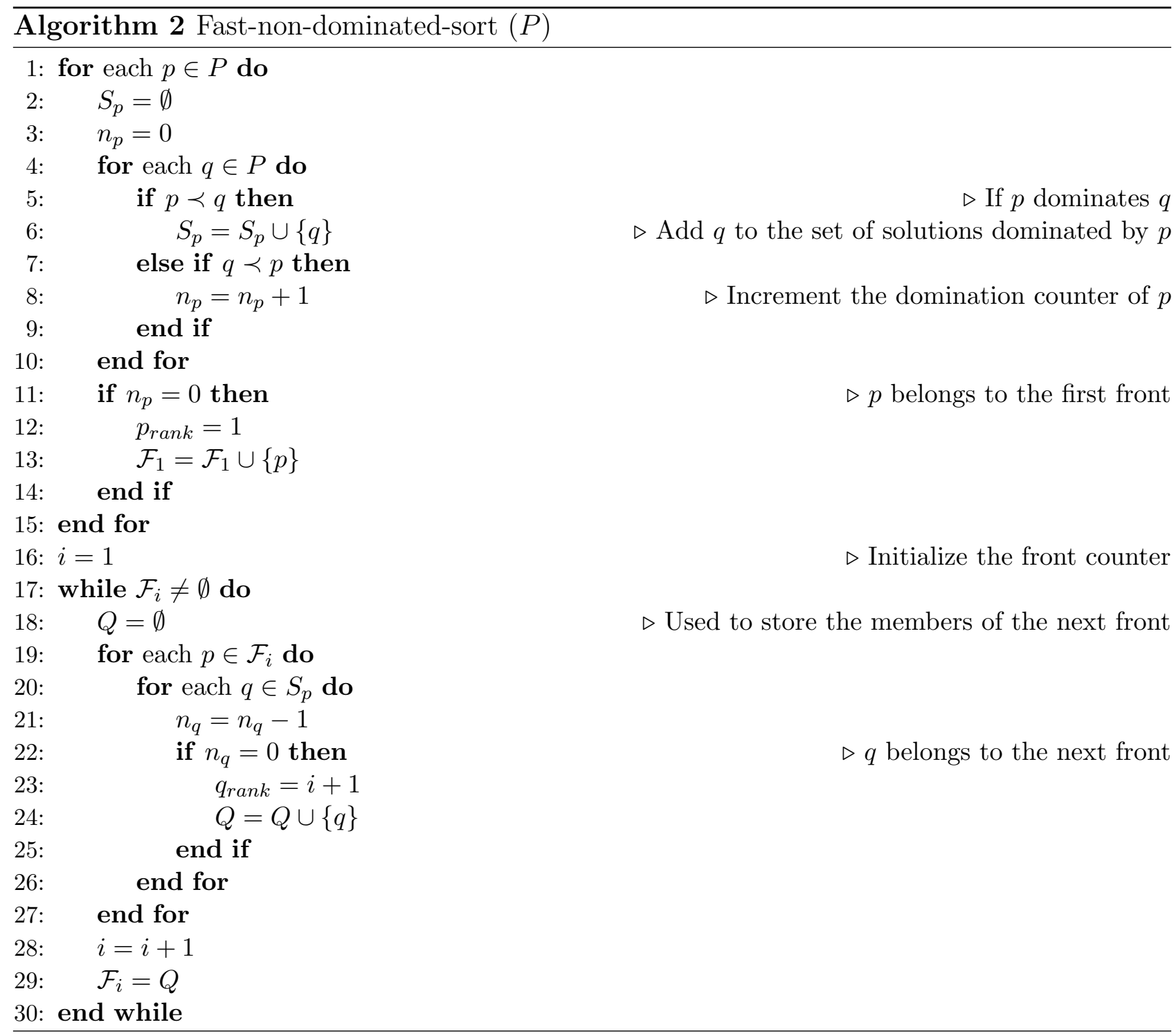




\section{Appendix A.2. Fast crowding distance estimation procedure}

In the proposed NSGA-II, the sharing function approach that the original NSGA used is replaced with a crowded-comparison approach which no longer requires any user-defined parameter for maintaining sustainable diversity among population members and has a better computational complexity.

To describe this approach, a density-estimation metric is firstly defined to get an estimate of the density of individuals surrounding a particular individual in the population. We calculate the average distance of two points on either side of this point along each of the objectives. This quantity $i_{\text {distance }}$ serves as an estimate of the perimeter of the cuboid formed by using the nearest neighbours as the vertices (called the crowding distance). The Algorithm 3 outlines the crowding distance computation procedure of all individuals in a nondominated set $I$ and has $O(m N \log N)$ computational complexity.

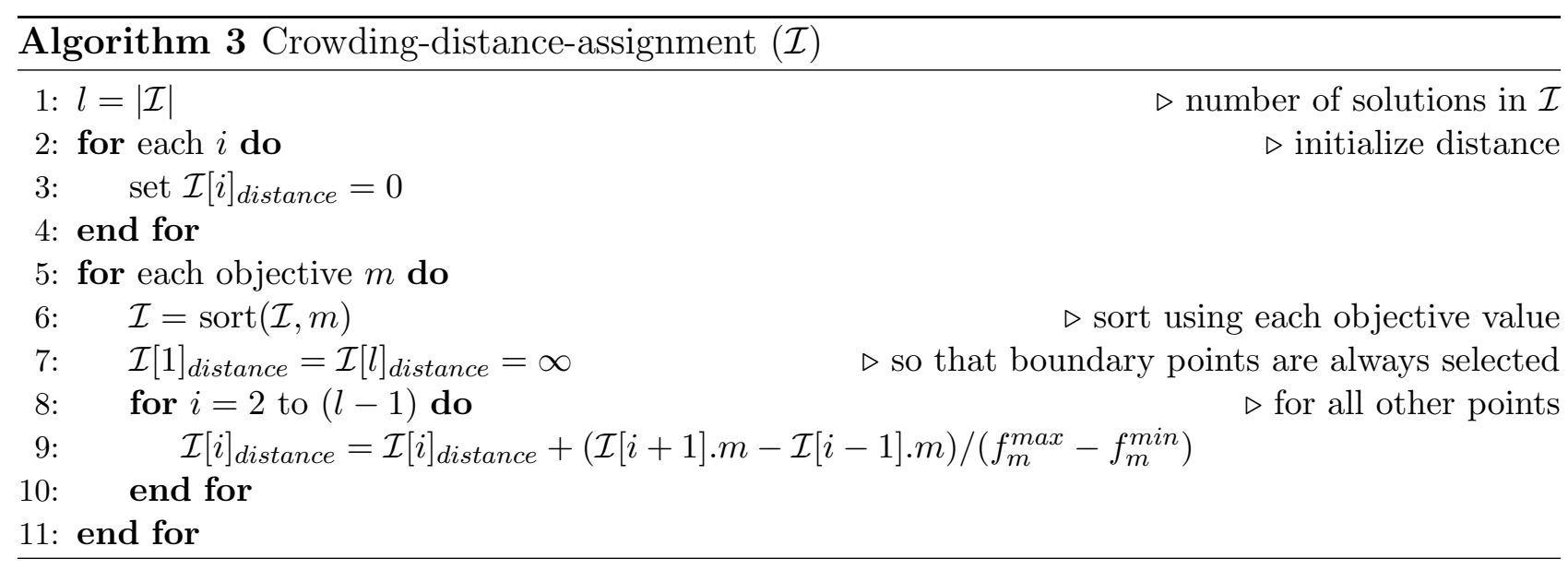

\section{Appendix A.3. Simple crowded-comparison operator}

The crowded-comparison operator $\left(\prec_{n}\right)$ guides the selection and elitism procedure at various stages of the algorithm to a uniformly spread-out Pareto-optimal front. In the selection step of this algorithm, we use a binary tournament selection based on crowded-comparison operator. Furthermore, in the elitist strategy, we utilise crowded-comparison operator to reduce the population. Each individual $i$ in the population has two attributes: (1) nondomination

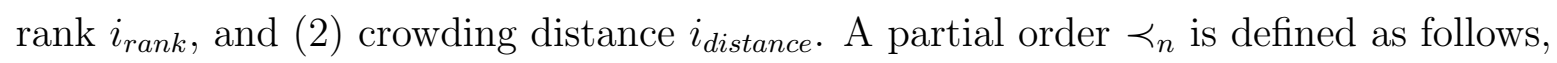

$$
\begin{aligned}
& i \prec_{n} j \quad \text { if }\left(i_{\text {rank }}<j_{\text {rank }}\right) \\
& \text { or }\left(\left(i_{\text {rank }}=j_{\text {rank }}\right) \text { and }\left(i_{\text {distance }}>j_{\text {distance }}\right)\right) .
\end{aligned}
$$

The individual with a lower rank is preferred between two individuals with different nondomination ranks or, if both individuals belong to the same front, we prefer the individual that 
is located in a less crowded region. The complexity of sorting on crowded-comparison operator is $O(N \log N)$.

\section{Appendix A.4. Crossover and mutation operator}

As this algorithm is based on real coding, it uses simulated binary crossover (SBX) operator for crossover process and polynomial mutation for mutation process. Distribution indexes $\eta_{c}$ and $\eta_{m}$ are used for crossover and mutation operators [13].

\section{Appendix A.5. Elitist strategy}

Elitism is to ensure that the excellent individuals in parent population can be selected to form the new parent population. It can speed up the performance of the GA significantly, which can also help in preventing the loss of good individuals once they are found. It needs to compare current population with the previously found best nondominated individuals, so we first describe the th generation of the proposed Algorithm 4 .

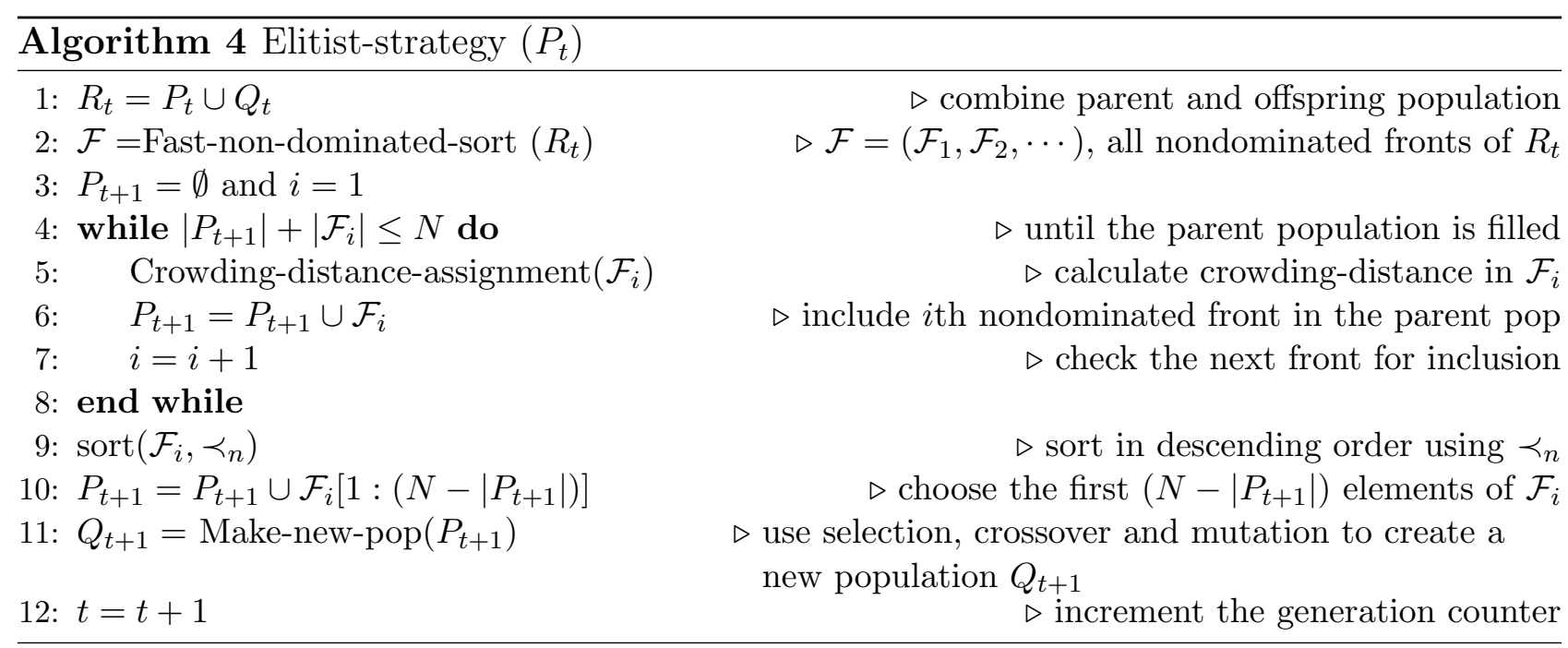

The new parent population $P_{t+1}$ of size $N$ is now used in the next generation or cycle for selection, crossover and mutation to create a new offspring population of size $N$.

Until now, the whole cycle of NSGA-II has been introduced. The overall computational complexity of the algorithm is $O\left(m N^{2}\right)$, which is up to the nondominated sorting procedure of the algorithm. The fast nondominated sorting procedure, the fast crowding distance estimation procedure, and the simple crowded-comparison operator are regarded as three innovations of NSGA-II, where the weaknesses of NSGA have been alleviated to a large extent owing to the improvements they brought in aspects of computational complexity, elitism and diversity preservation. Based on the previous literature, we conclude a complete process of this algorithm given in Fig. A.6. 


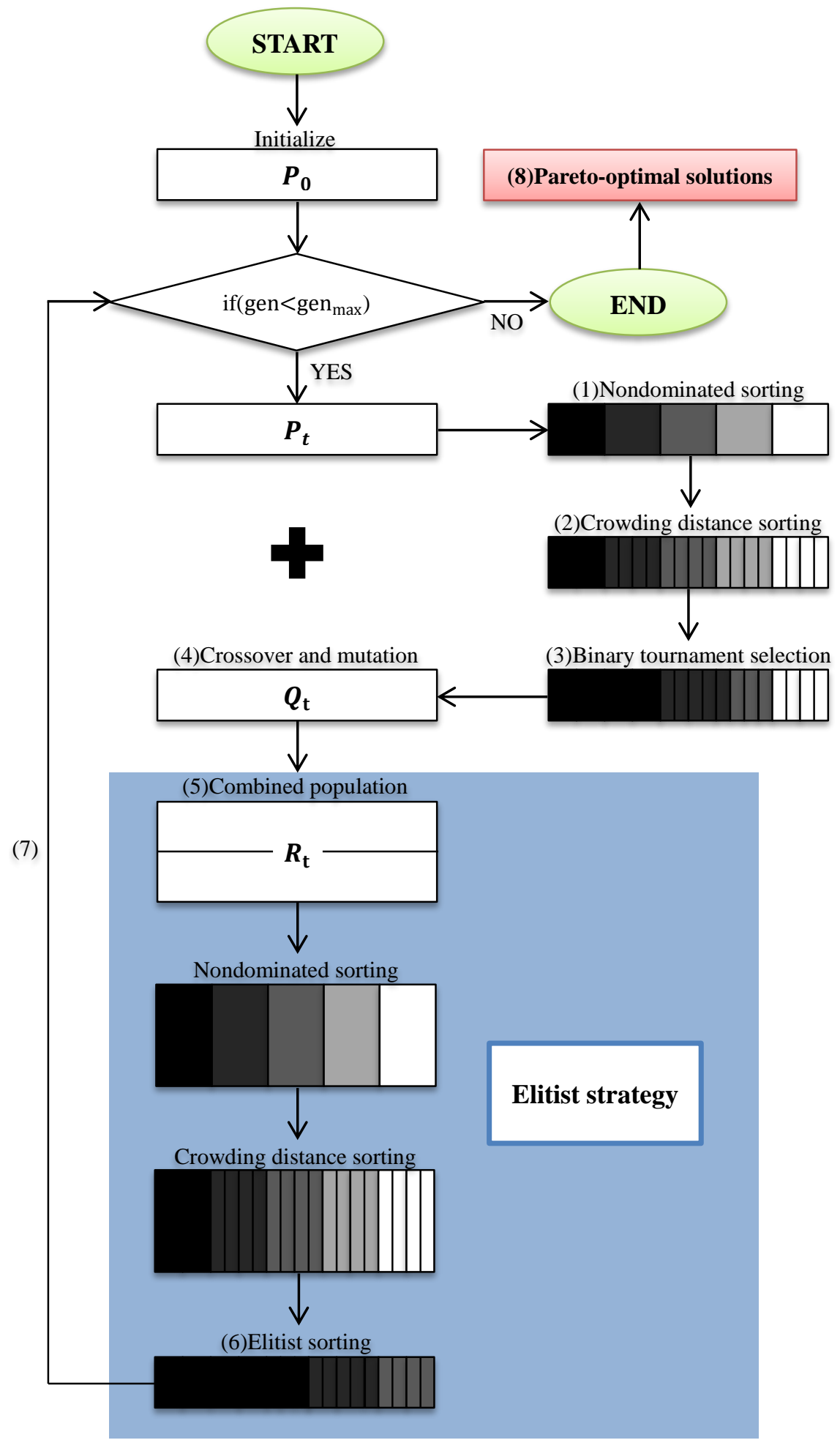

Fig. A.6: Process of NSGA-II.

Appendix B. Parameter settings of the model for the numerical example 


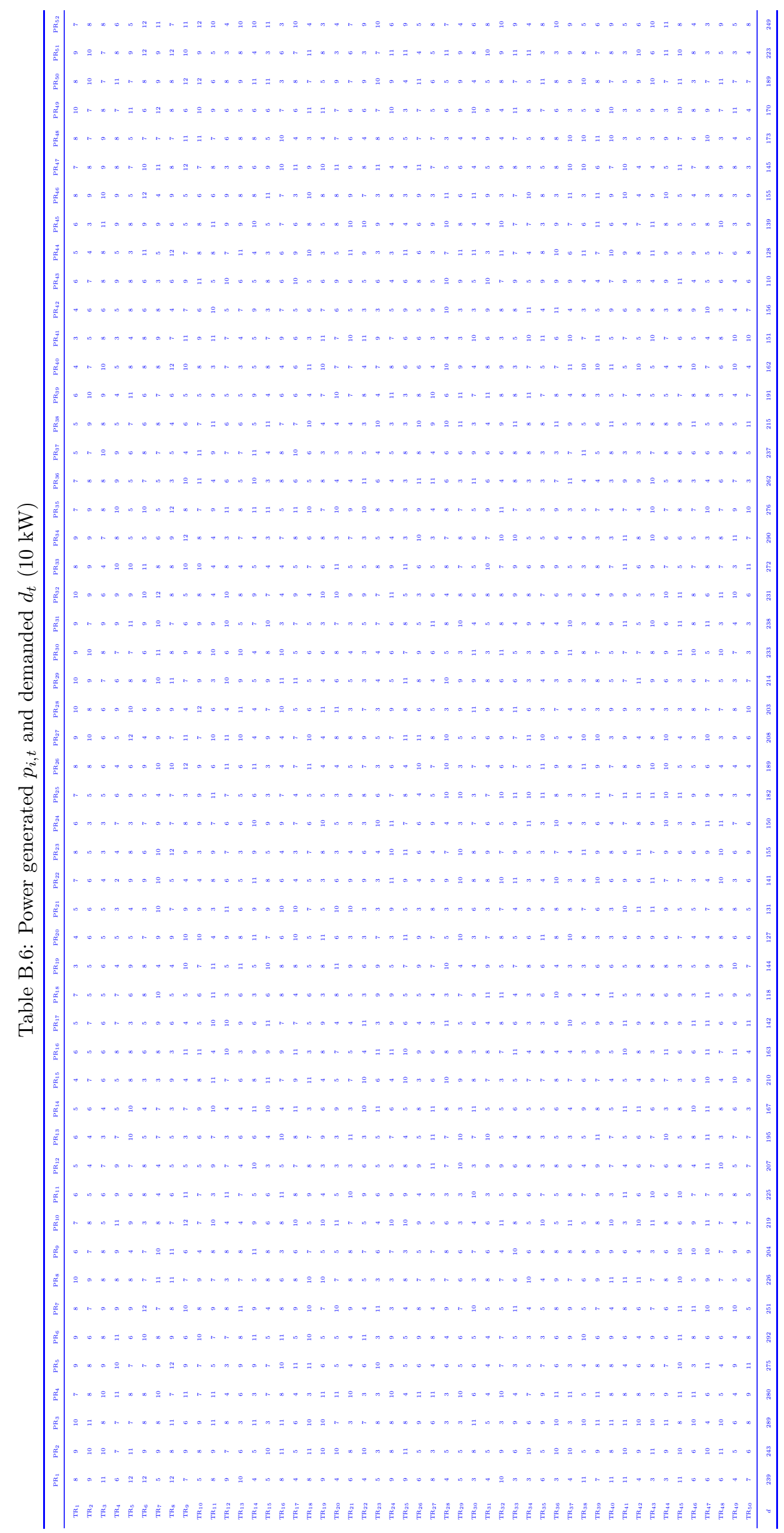




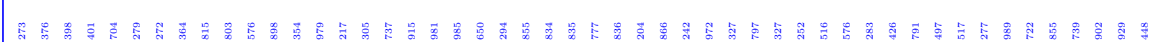

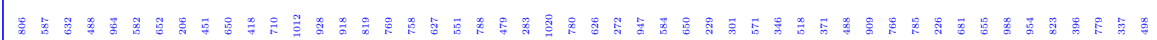

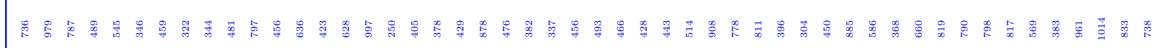

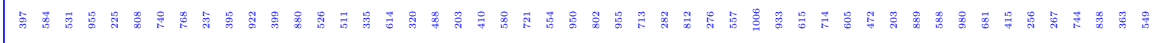

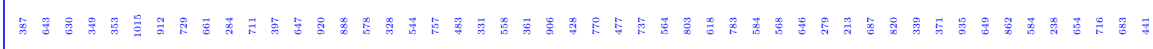

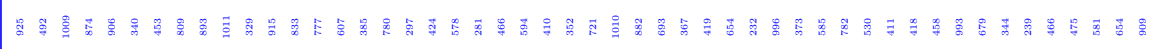

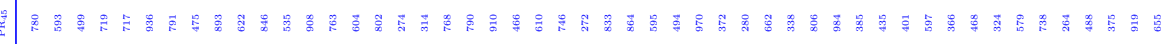

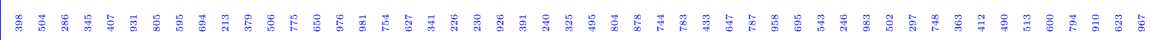

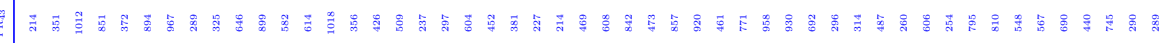

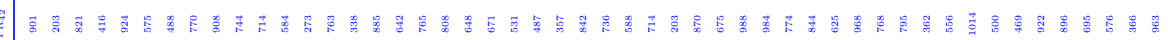

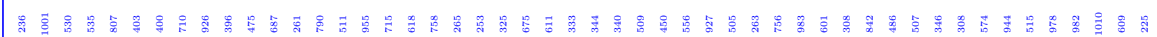

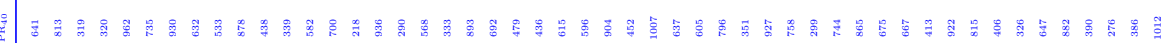

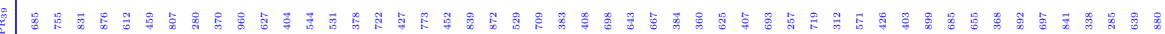

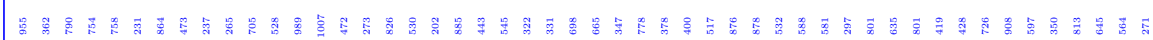

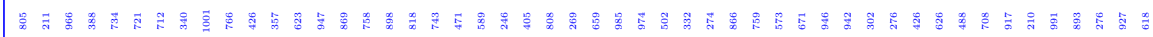

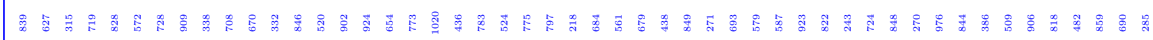

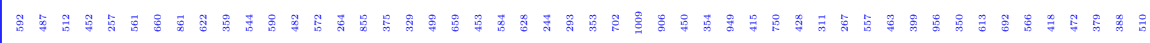

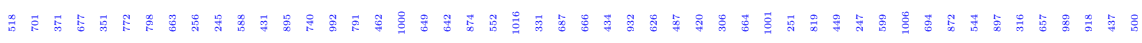

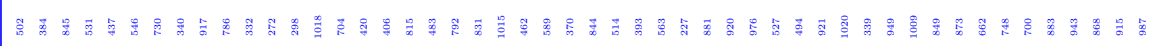

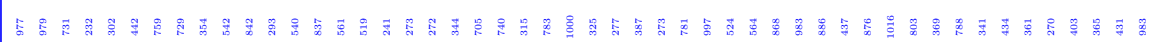

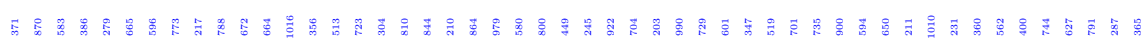

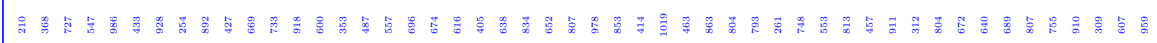

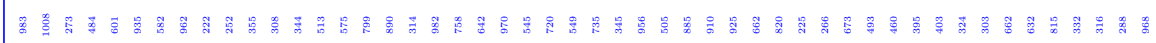

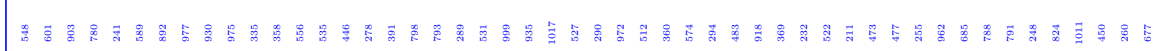

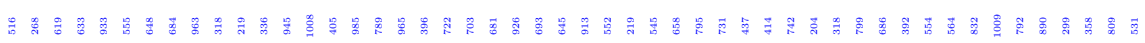

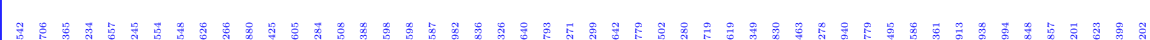

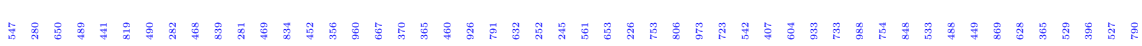

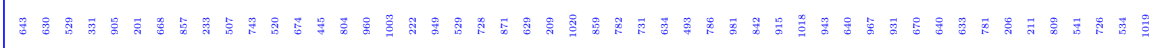

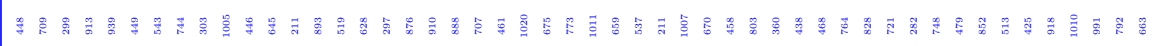

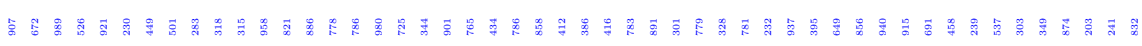

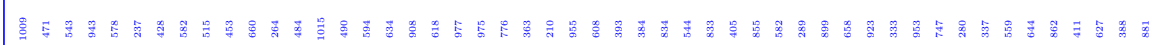

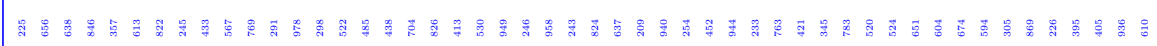

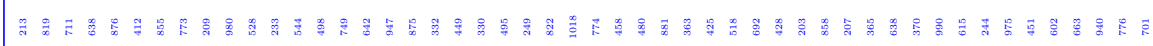

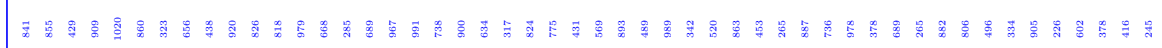

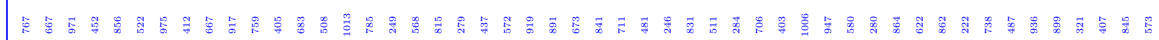

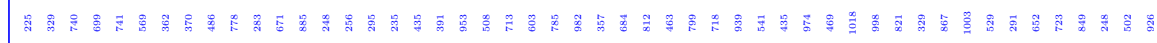

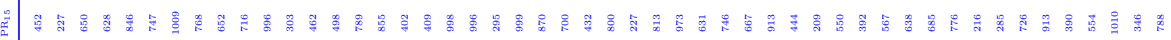

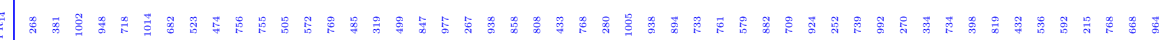

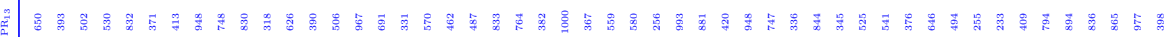

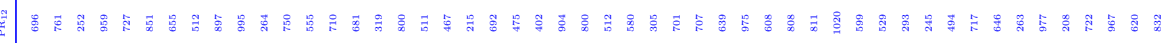

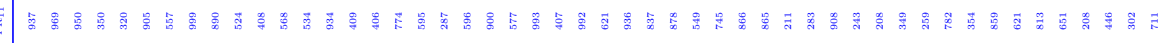

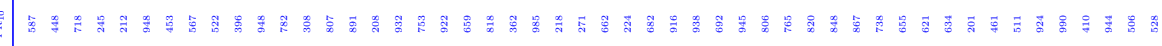

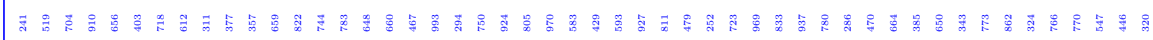

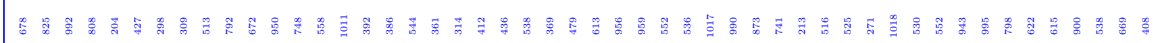

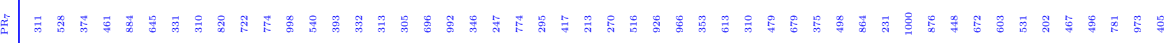

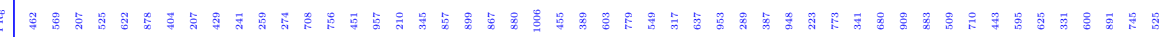

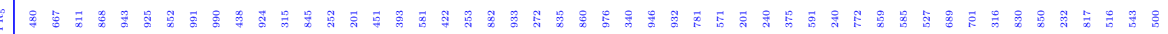

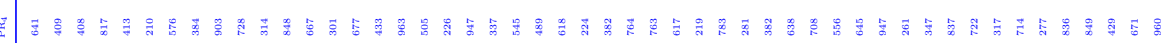

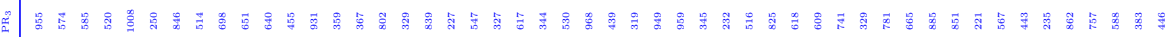

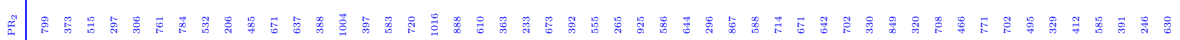

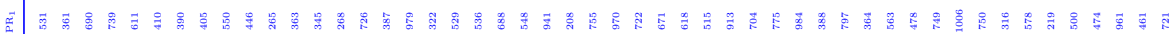

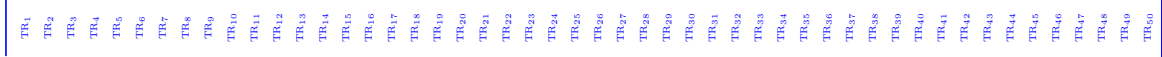




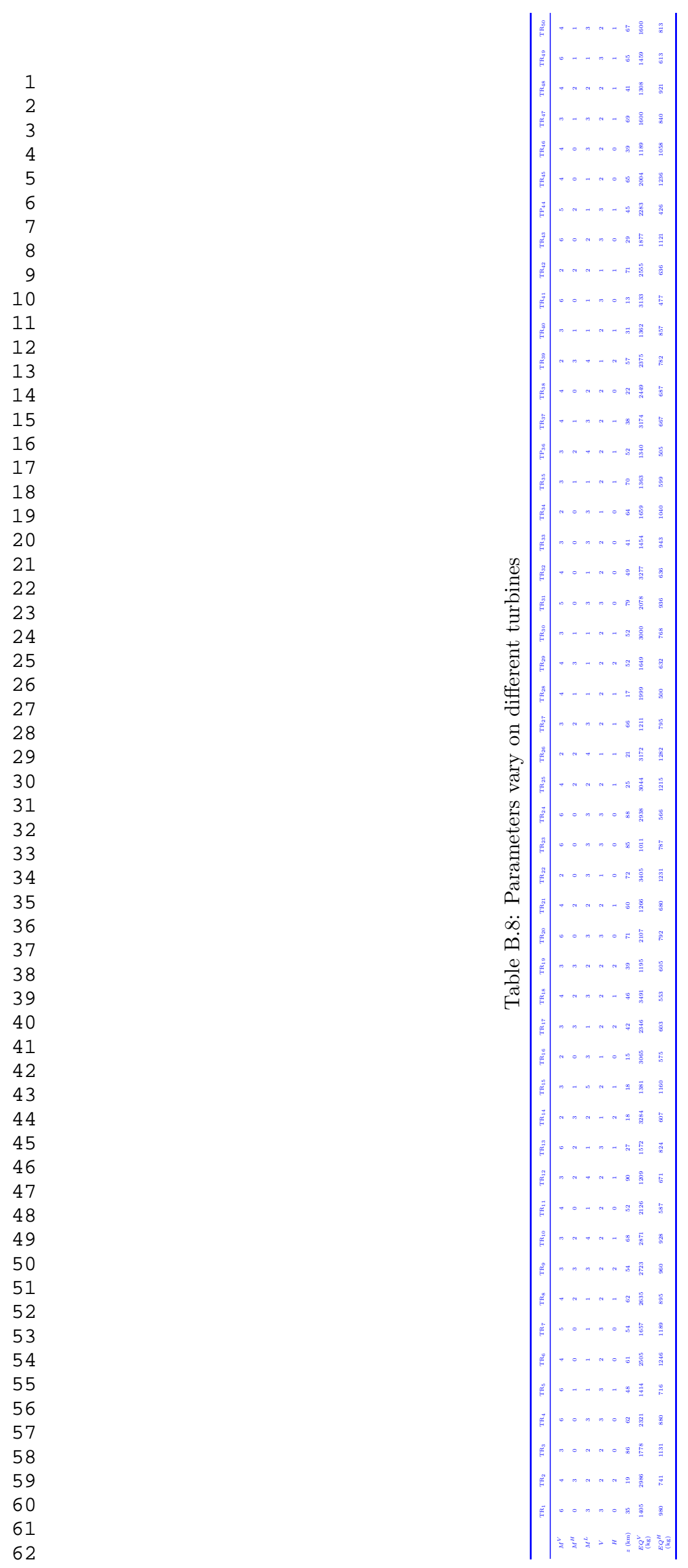




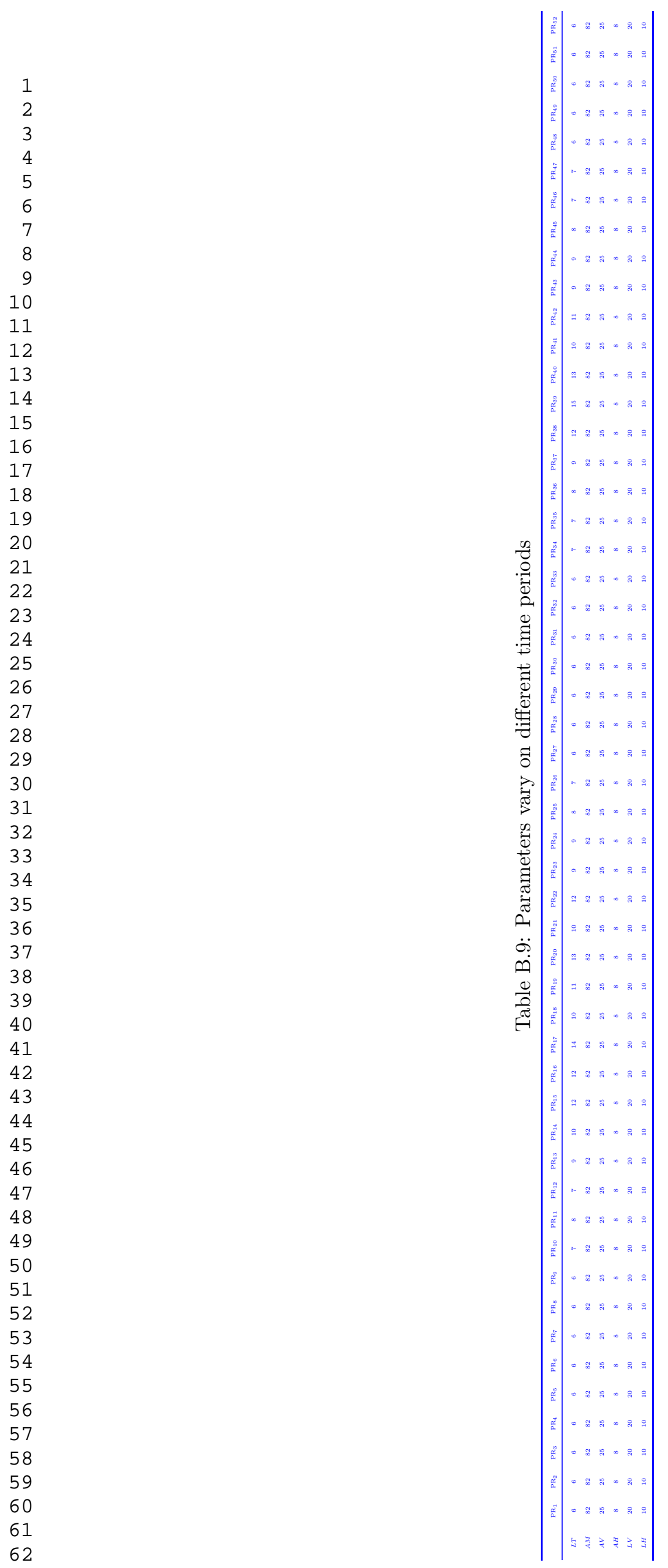


Table B.10: Remaining parameters

\begin{tabular}{ll}
\hline Model parameter & Parameter value \\
\hline$q^{V}(\mathrm{~kg} / \mathrm{kg} \cdot \mathrm{km})$ & $10^{-5}$ \\
$q^{H}(\mathrm{~kg} / \mathrm{kg} \cdot \mathrm{km})$ & $2 \times 10^{-4}$ \\
$w(\mathrm{~kg})$ & 65 \\
$G H G(\mathrm{~kg})$ & 110 \\
\hline
\end{tabular}

Table B.11: Parameters for NSGA-II

\begin{tabular}{ll}
\hline NSGA-II parameter & Parameter value (type) \\
\hline Population size, $N$ & 100 \\
Length of individual, $M$ & 50 \\
Number of maximum generations, maxgen & 5000 \\
Crossover probability, $p_{c}$ & 0.54 \\
Mutation probability, $p_{m}$ & 0.06 \\
Crossover index, $\eta_{c}$ & 20 (simulated binary crossover) \\
Mutation index, $\eta_{m}$ & 20 (polynomial mutation) \\
\hline
\end{tabular}

